Research Article

\title{
Trajectory Tracking Control Study of a New Parallel Mechanism with Redundant Actuation
}

\author{
Haiqiang Zhang $\mathbb{D}^{1,2}$ Hairong Fang ${ }^{1},{ }^{1}$ Dan Zhang, ${ }^{2}$ Qi Zou, ${ }^{2}$ and Xueling Luo ${ }^{2}$ \\ ${ }^{1}$ School of Mechanical, Electronic and Control Engineering, Beijing Jiaotong University, Beijing, China \\ ${ }^{2}$ Lassonde School of Engineering, York University, ON, Canada M3J 1P3 \\ Correspondence should be addressed to Haiqiang Zhang; haiqiang@yorku.ca and Hairong Fang; hrfang@bjtu.edu.cn
}

Received 17 June 2019; Revised 8 December 2019; Accepted 28 December 2019; Published 11 January 2020

Academic Editor: Linda L. Vahala

Copyright ( 2020 Haiqiang Zhang et al. This is an open access article distributed under the Creative Commons Attribution License, which permits unrestricted use, distribution, and reproduction in any medium, provided the original work is properly cited.

\begin{abstract}
Parallel mechanisms with redundant actuation are attracting numerous scholars' research interest due to their inherent advantages. In this paper, an efficient trajectory tracking control scheme for the new redundantly actuated parallel mechanism by integrating force/position hybrid control with the combination of inertia feed-forward control and back propagation (BP) neural network PID control is proposed. The dynamic models including the joint space and task space are formulated explicitly in efficient and compact form by means of the principle of virtual work and d'Alembert formulations. The force/position hybrid control is implemented to perform trajectory tracking and optimize the driving force configuration in MATLAB/Simulink environment, before being applied to an actual parallel mechanism. The illustrative simulation results demonstrate that the force/position hybrid control scheme is available to provide good trajectory tracking performance. Simultaneously, the feasibility of the proposed control scheme is verified by comparison analysis with the aforementioned conventional control method.
\end{abstract}

\section{Introduction}

Compared to counterpart serial mechanisms, parallel mechanisms exhibit much greater advantages such as high stiffness, lower inertia, high loading capability, high precision, large acceleration, and high dexterity, which are desirable properties for use in high speed machine, high precision assisted surgery, high speed pick and place of delta robots, and some other applications [1-3]. However, small workspace and many singularities within the workspace limit their wide applications. Under such circumstance, parallel mechanisms with redundant actuation are expected to tackle their shortcomings, as redundant actuation can eliminate or decrease singularities, increase the dexterous workspace, optimize the configuration of driving force, and so on. At present, the research works on redundant actuation are mainly focused on the singularities and the workspace. However, the intelligent control strategies in the existing literature are seldom studied [4-6], which has just gotten more attention to make the most use of their advantages; in particular, for high speed, high accuracy machine tools, trajectory track- ing control, for instance, adaptive control and robust control, is very essential for more prosperous applications [7-11].

Redundant actuation can be achieved in a different method; one is to add the kinematic chain including nonconstrained subchains or constrained subchains without increasing the degree of freedom, and the other is to change the passive joint into active joint [12]. Gosselin and Schreiber [13] increased a kinematically redundant leg in order to preserve the force transmission properties of the well-known Gough-Stewart parallel mechanism, which can avoid singularities and extend the rotational workspace. Liu et al. [14] studied exhaustively the force/motion transmissibility of redundantly actuated parallel mechanisms, and six simulation examples illustrate that the actuation redundancy can significantly improve the kinematic performance and enlarge the singularity-free workspace. Yao et al. [15] presented a redundant actuated 5UPS-PRPU parallel mechanism by adding an actuator for the middle PRPU passive constraint branch; the simulations illustrated that redundant actuation can greatly improve the performance and ability of the parallel mechanism. Boudreau et al. $[16,17]$ presented the dynamic 
model of a cinematically redundant planar parallel mechanism and an optimization to minimize the torques when the end-effector performs the trajectory tracking. Wang et al. [18] derived the inverse dynamic model of a spatial 3DOF parallel mechanism with redundant actuation by adding actuators to passive rotational joints, and the driving force was optimized in terms of the Moore-Penrose inverse matrix. The motion control is very complicated for the coupled dynamics of the parallel mechanism with redundant actuation. Wen et al. [19] investigated a new hybrid control algorithm for the redundantly actuated 6PUS-UPU parallel robot, i.e., the inner model control in the position loop and the fractional order fuzzy proportional-integral derivative control in the force loop. In addition, in [20], two model predictive controllers including proportional, integral, and differential algorithm and fuzzy algorithm were proposed for a five-degree-of-freedom redundantly actuated parallel mechanism. And the simulation results showed that the fuzzy model predictive control can effectively improve real-time performance of the control system compared with the traditional PID model predictive control algorithm. Liu et al. [21] proposed a model-based driving force synchronous control method with neural network, which can effectively reduce the driving force errors of the parallel mechanism. Similarly, Luces et al. [22] presented an in-depth discussion of past research on parallel mechanism kinematic redundancy and indicated that primary advantages of the parallel mechanism with redundant actuation contain workspace enlargement, singularity elimination/avoidance, and joint-torque optimum distribution, yet redundant actuation is facing a valuable opportunity as well as great challenges, such as motion planning and control and calibration. Flohic et al. [23] adopted a hybrid force/position control algorithm to conduct parallel kinematic machine for mechanical testing, and the control algorithm is illustrated on a Nooru-Mohammed test performed on a Gough-Stewart platform and validated experimentally on a five-bar parallel mechanism. Cazalilla et al. $[24,25]$ developed an adaptive controller for the 3-PRS parallel mechanism with consideration of some uncertainties; the simulation results show that the adaptive controller can greatly improve the trajectory tracking precision compared with the passivity-based controllers. Urrea and Kern [26] presented a summary of the controllers considering for the evaluation of the redundant robot model with their actuators and corresponding performance. Callegari et al. [27] presented the inverse dynamic model by means of the principle of virtual work; three control strategies were employed and the results show that hybrid position/force schemes should be able to provide better performance than the other position control or force control. Achievements that are both important and probably of a very scientific perspective were achieved by Siciliano in [28]; in these recent works, they took profit from a general solution for the inverse dynamic of parallel mechanism with redundant actuation to construct a computationally efficient control scheme compared with a conventional control one, which makes it available in control applications with singularity avoidance. In this paper, the conventional PID control algorithm is unavailable to be implemented on the proposed parallel mechanism,

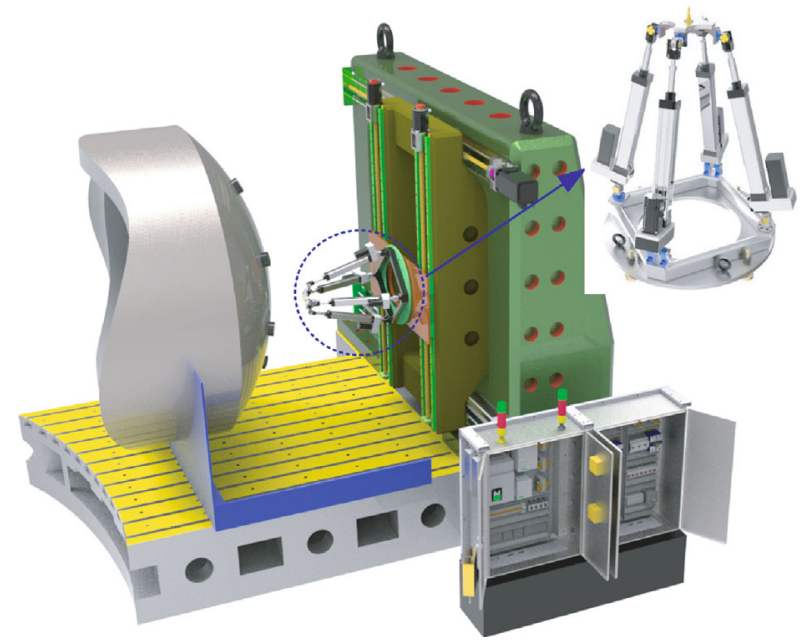

FIgure 1: A five-axis hybrid kinematic machine tool.

so the force/position hybrid control algorithm is carried out on the redundantly actuated parallel mechanism to perform trajectory tracking.

The remainder of this paper is organized as follows: Section 2 describes the structure of the redundantly actuated 2RPU-2SPR parallel mechanism and the coordinate frames. The kinematic relations of the parallel mechanism are developed in detail in Section 3. In Section 4, the explicit dynamic model is derived in terms of the principle of virtual work and d'Alembert formulation. Next, in Section 5, the force/position hybrid control algorithm is presented. In Section 6, a force/position hybrid control simulation case is given. Finally, some brief contributions and future works are presented.

\section{System Description}

The serial-parallel hybrid kinematic machine (HKM) structure was selected as a candidate architecture for complicated surface milling in aerospace domain [29] (as shown in Figure 1), whose parallel module is a $2 \mathrm{RPU}-2 \mathrm{SPR}$ parallel mechanism with redundant actuation $(R, P, \bar{U}$, and $S$ standing for rotational, prismatic, universal, and spherical kinematic joint, respectively). The underline format $(\underline{P})$ represents the actuated joint. The structural diagram is depicted in Figure 2. The new parallel mechanism mainly consists of the fixed platform, the moving platform, and four limbs with two of the same identical kinematic configuration. The prismatic joints described by the linear joint variables $d_{i}$ connects the lower platform to the upper platform by a rotational joint followed by a universal joint or a spherical joint followed by a rotational joint. It is worth noting that the used spherical joint is designed by means of three mutually perpendicular rotational joints so as to enlarge the rotation range.

For modeling purposes, as shown in Figure 2, we assume a fixed coordinate system $B-x y z$ at the centered point $B$ of the fixed platform and a moving coordinate system $A-u v w$ on the square moving platform at the centered point $A$, along with the $z$-axis and $w$-axis perpendicular to the platform, and the $x$-axis and $y$-axis parallel to the $u$-axis and $v$-axis, respectively. Both $A_{1} A_{2} A_{3} A_{4}$ and $B_{1} B_{2} B_{3} B_{4}$ are designed to be 


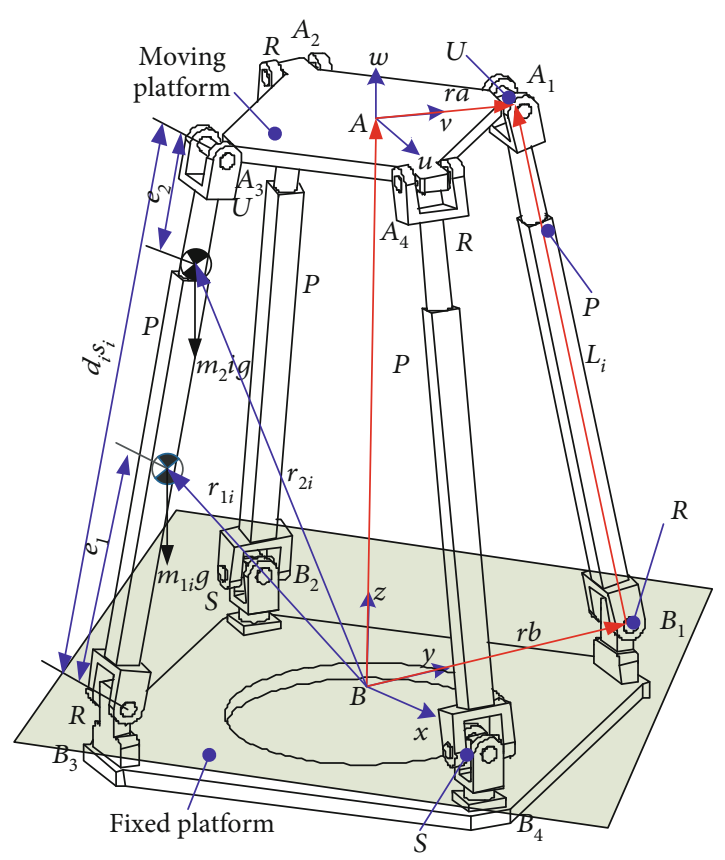

FIGURE 2: Schematic diagram of the 2RPU-2SPR parallel mechanism.

squares so as to yield a symmetric architecture of the parallel mechanism. With these geometric conditions satisfied, the moving platform of parallel mechanism will pose with three independent degrees of freedom in the Cartesian space by controlling the movement of four servo actuators, that is, two rotational degrees of freedom around the $x$-axis and $v$-axis and one translational degree of freedom along the $z$-axis; in this case, the spatial rotations are coupled with the change of four nonidentical limbs [30]. What is more, as the number of actuated joints is greater than that of the degree of freedom, it belongs to a redundantly actuated parallel mechanism. Nevertheless, if the fourth limb, i.e., the SPR limb, is removed, the parallel mechanism will turn into a general nonredundant parallel mechanism.

\section{Kinematic Analysis}

The moving platform can move along the $z$ direction and rotate around the $x$-axis and $v$-axis with the consideration of mobility characteristics. The homogeneous transformation matrix $\mathbf{T}$ of the reference coordinate point $A$ can be expressed as follows:

$$
\begin{aligned}
\mathbf{T} & =\left[\begin{array}{ll}
\mathbf{R} & \mathbf{p} \\
0 & 1
\end{array}\right]=\operatorname{Rot}(x, \alpha) \operatorname{Trans}(z, d) \operatorname{Rot}(v, \beta) \\
& =\left[\begin{array}{cccc}
\cos \beta & 0 & \sin \beta & x \\
\sin \alpha \sin \beta & \cos \alpha & -\sin \alpha \cos \beta & y \\
-\sin \beta \cos \alpha & \sin \alpha & \cos \alpha \cos \beta & z \\
0 & 0 & 0 & 1
\end{array}\right],
\end{aligned}
$$

where $\mathbf{p}=[x, y, z]^{T}$ is the position coordinates of point $A$ in the fixed coordinate system, $\alpha$ is the rotation angle around the $x$-axis, and $\beta$ is the rotation angle around the $v$-axis.

Additionally, once the rotation matrix $\mathbf{R}$ is obtained, then the rotational Euler angles will be obtained by the following (2), which are important for achieving the orientation angles in the feedback control proposed in this paper:

$$
\left\{\begin{array}{l}
\alpha=\arctan 2(\mathbf{R}(3,2), \mathbf{R}(2,2)), \\
\beta=\arctan 2(\mathbf{R}(1,3), \mathbf{R}(1,1)),
\end{array}\right.
$$

where $\mathbf{R}(\cdot, \cdot)$ represents the row and column elements of the rotation matrix $\mathbf{R}$.

The inverse Jacobian matrix relationship between input and output can be represented as follows:

$$
\dot{\mathbf{q}}=\mathbf{J}_{0} \dot{\mathbf{X}}=\mathbf{J}_{q} \mathbf{J}_{p} \dot{\mathbf{X}},
$$

where $\dot{\mathbf{q}}=\left[\dot{d}_{1}, \dot{d}_{2}, \dot{d}_{3}, \dot{d}_{4}\right]^{T}, \mathbf{J}_{0}$ is the velocity Jacobian matrix, and

$$
\begin{aligned}
& \mathbf{J}_{q}= {\left[\begin{array}{ll}
\mathbf{s}_{1}^{T} & {\left[\mathbf{a}_{1} \times \mathbf{s}_{1}\right]^{T}} \\
\mathbf{s}_{2}^{T} & {\left[\mathbf{a}_{2} \times \mathbf{s}_{2}\right]^{T}} \\
\mathbf{s}_{3}^{T} & {\left[\mathbf{a}_{3} \times \mathbf{s}_{3}\right]^{T}} \\
\mathbf{s}_{4}^{T} & {\left[\mathbf{a}_{4} \times \mathbf{s}_{4}\right]^{T}}
\end{array}\right], } \\
& \mathbf{J}_{p}=\left[\begin{array}{c}
\mathbf{J}_{v p} \\
\mathbf{J}_{w p}
\end{array}\right]=\left[\begin{array}{ccc}
0 & 0 & 0 \\
-\tan \alpha & -z \cdot \sec ^{2} \alpha & 0 \\
1 & 0 & 0 \\
0 & 1 & 0 \\
0 & 0 & \cos \alpha \\
0 & 0 & \sin \alpha
\end{array}\right],
\end{aligned}
$$

where $\mathbf{s}_{i}$ denotes the direction vector of $i$-th $\operatorname{limb}, \mathbf{a}_{i}=\mathbf{R} \cdot{ }^{A} \mathbf{a}_{i}$, ${ }^{A} \mathbf{a}_{i}$ is the description of the position vector in the moving coordinate system, and $\mathbf{X}=\left[\begin{array}{lll}z & \alpha & \beta\end{array}\right]^{T}$ is the independent motion degree of freedom parameters.

By differentiating both sides of (3), the acceleration between the actuated joints and corresponding end-effector can be expressed as

$$
\ddot{\mathbf{q}}=\dot{\mathbf{J}}_{0} \dot{\mathbf{X}}+\mathbf{J}_{0} \ddot{\mathbf{X}}
$$

where 


$$
\begin{aligned}
& \dot{\mathbf{J}}_{0}=\left[\begin{array}{cc}
{\left[\mathbf{w}_{1} \times \mathbf{s}_{1}\right]^{T}} & {\left[\left(\mathbf{w}_{p} \times \mathbf{a}_{1}\right) \times \mathbf{s}_{1}+\mathbf{a}_{1} \times\left(\mathbf{w}_{1} \times \mathbf{s}_{1}\right)\right]^{T}} \\
{\left[\mathbf{w}_{2} \times \mathbf{s}_{2}\right]^{T}} & {\left[\left(\mathbf{w}_{p} \times \mathbf{a}_{2}\right) \times \mathbf{s}_{2}+\mathbf{a}_{2} \times\left(\mathbf{w}_{2} \times \mathbf{s}_{2}\right)\right]^{T}} \\
{\left[\mathbf{w}_{3} \times \mathbf{s}_{3}\right]^{T}} & {\left[\left(\mathbf{w}_{p} \times \mathbf{a}_{3}\right) \times \mathbf{s}_{3}+\mathbf{a}_{3} \times\left(\mathbf{w}_{3} \times \mathbf{s}_{3}\right)\right]^{T}} \\
{\left[\mathbf{w}_{4} \times \mathbf{s}_{4}\right]^{T}} & {\left[\left(\mathbf{w}_{p} \times \mathbf{a}_{4}\right) \times \mathbf{s}_{4}+\mathbf{a}_{4} \times\left(\mathbf{w}_{4} \times \mathbf{s}_{4}\right)\right]^{T}}
\end{array}\right] \cdot \mathbf{J}_{p} \\
& {\left[\begin{array}{ccc}
0 & 0 & 0 \\
\left(-1-\tan ^{2} \alpha\right) \cdot \dot{\alpha} & -\left(\dot{z} \cdot \sec ^{2} \alpha+\frac{2 z \cdot \sin \alpha}{\cos ^{3} \alpha} \cdot \dot{\alpha}\right) & 0 \\
0 & 0 & 0 \\
0 & 0 & 0 \\
0 & 0 & -\sin \alpha \cdot \dot{\alpha} \\
0 & 0 & \cos \alpha \cdot \dot{\alpha}
\end{array}\right] \text {, }}
\end{aligned}
$$

where $\mathbf{w}_{i}$ is the angular velocity of the $i$-th limb defined in the fixed coordinate system.

The determination of $\dot{\mathbf{J}}_{0}$ is actually very important for the next section about dynamic analysis.

It is analogous to (3); the forward Jacobian matrix $\mathbf{J}_{f}$ which characterized the relationship between the end-effector and corresponding actuating joints can be expressed as

$$
\dot{\mathbf{X}}=\mathbf{J}_{f} \dot{\mathbf{q}}, \quad \mathbf{J}_{f}=\mathbf{J}_{0}{ }^{+},
$$

where $[\cdot]^{+}$denotes the Moore-Penrose inverse matrix. The Moore-Penrose inverse of the matrix $\mathbf{J}_{0}$ can be denoted as $\left(\mathbf{J}_{0}\right)^{+}=\mathbf{J}_{0}^{T}\left(\mathbf{J}_{0} \mathbf{J}_{0}^{T}\right)^{-1}$ and the condition $\mathbf{J}_{0} \mathbf{J}_{0}{ }^{+}=\mathbf{I}$ should be satisfied.

Hence, the following relationship should be satisfied:

$$
\mathbf{J}_{f} \cdot \mathbf{J}_{0}=\mathbf{J}_{0} \cdot \mathbf{J}_{f}
$$

Taking the derivative of (8) with respect to time, yield

$$
\begin{aligned}
\frac{d}{d t}\left(\mathbf{J}_{f} \cdot \mathbf{J}_{0}\right) & =0 \Rightarrow \dot{\mathbf{J}}_{f} \cdot \mathbf{J}_{0}+\mathbf{J}_{f} \cdot \dot{\mathbf{J}}_{0}=0 \Rightarrow \dot{\mathbf{J}}_{f} \\
& =-\mathbf{J}_{0}{ }^{-1} \cdot \dot{\mathbf{J}}_{0} \cdot \mathbf{J}_{0}{ }^{-1} .
\end{aligned}
$$

The structural diagram of the $i$-th limb is depicted in Figure 2; each of that consists of an upper sublimb and a lower sublimb. Let $e_{1}$ be the distance between the centroid $B_{i}$ and the center of mass of the lower sublimb, while $e_{2}$ be the distance of the centroid $A_{i}$ and the center of mass of the upper sublimb. The position vectors of the centers of mass of the $i$-th limb are given with respect to the fixed coordinate system:

$$
\begin{aligned}
& \mathbf{r}_{1 i}=\mathbf{b}_{i}+e_{1} \mathbf{s}_{i}, \\
& \mathbf{r}_{2 i}=\mathbf{b}_{i}+\left(d_{i}-e_{2}\right) \mathbf{s}_{i} .
\end{aligned}
$$

The inverse kinematics should be differentiated with respect to time, and the velocity of kinematic joints $A_{i}$ connected to the moving platform can be obtained in the fixed coordinate system:

$$
\mathbf{v}_{a i}=\mathbf{v}_{p}+\omega_{p} \times \mathbf{a}_{i}=\mathbf{J}_{a i} \cdot \mathbf{J}_{p} \cdot \dot{\mathbf{X}}
$$

where

$$
\mathbf{J}_{a i}=\left[\begin{array}{cccccc}
1 & 0 & 0 & 0 & a_{i z} & -a_{i y} \\
0 & 1 & 0 & -a_{i z} & 0 & a_{i x} \\
0 & 0 & 1 & a_{i y} & -a_{i x} & 0
\end{array}\right]
$$

The angular velocity of the $i$-th limb

$$
\omega_{i}=\frac{\mathbf{s}_{i} \times \mathbf{v}_{a i}}{d_{i}}=\mathbf{J}_{w i} \dot{\mathbf{X}},
$$

where $\mathbf{J}_{w i}=\tilde{\mathbf{s}}_{i} \mathbf{J}_{a i} \mathbf{J}_{p} / d_{i}$, and $\tilde{\mathbf{s}}_{i}$ is antisymmetric operator corresponding to the vector $\mathbf{s}_{i}$.

Taking the derivative of (10) and (11) with respect to time, the velocity of the center of mass of the lower sublimb and upper sublimb can be obtained in the fixed coordinate system.

$$
\mathbf{v}_{1 i}=e_{1} \omega_{i} \times \mathbf{s}_{i}=\mathbf{J}_{v 1 i} \dot{\mathbf{X}}
$$

where $\mathbf{J}_{v 1 i}=-e_{1} \cdot \tilde{\mathbf{s}}_{i} \mathbf{J}_{w i}$

$$
\begin{aligned}
& \mathbf{v}_{2 i}=\left(d_{i}-e_{2}\right) \boldsymbol{\omega}_{i} \times \mathbf{s}_{i}+\dot{d}_{i} \cdot \mathbf{s}_{i}=\mathbf{J}_{v 2 i} \dot{\mathbf{X}} \\
& \mathbf{J}_{v 2 i}=\left(e_{2}-d_{i}\right) \cdot \tilde{\mathbf{s}}_{i} \mathbf{J}_{w i}+\mathbf{s}_{i} \mathbf{J}_{d i} \mathbf{J}_{p}
\end{aligned}
$$


Turning to accelerations, the acceleration of the point $A_{i}$ can be carried out by differentiating (12) with respect to time:

$$
\dot{\mathbf{v}}_{a i}=\dot{\mathbf{v}}_{p}+\dot{\omega}_{p} \times \mathbf{a}_{i}+\omega_{p} \times\left(\omega_{p} \times \mathbf{a}_{i}\right) .
$$

The angular acceleration velocity of the $i$-th limb can be expressed as

$$
\dot{\omega}_{i}=\frac{\mathbf{s}_{i} \times \dot{\mathbf{v}}_{a i}-2 \dot{d}_{i} \omega_{i}}{d_{i}}=\mathbf{J}_{w i} \ddot{\mathbf{X}}+\dot{\mathbf{J}}_{w i} \dot{\mathbf{X}}
$$

where

$\dot{\mathbf{J}}_{w i}=\frac{\tilde{\mathbf{s}}_{i}\left(\left(\dot{\mathbf{J}}_{v p}-\tilde{\mathbf{a}}_{i} \dot{\mathbf{J}}_{w p}\right)-\left[\left(\mathbf{J}_{w p} \dot{\mathbf{X}}\right) \times\right] \tilde{\mathbf{a}}_{i} \mathbf{J}_{w p}\right)-2 \mathbf{J}_{d i} \mathbf{J}_{0} \dot{\mathbf{X}} \mathbf{J}_{w i}}{d_{i}}$.

Finally, the acceleration of the centers of mass of the $i$-th limb can be obtained by differentiating once again (15) and (16) with respect to time:

$$
\dot{\mathbf{v}}_{1 i}=e_{1} \dot{\omega}_{i} \times \mathbf{s}_{i}+e_{1} \omega_{i} \times\left(\omega_{i} \times \mathbf{s}_{i}\right)=\mathbf{J}_{v 1 i} \ddot{\mathbf{X}}+\dot{\mathbf{J}}_{v 1 i} \dot{\mathbf{X}}
$$

where

$$
\begin{aligned}
\dot{\mathbf{J}}_{v 1 i}= & -e_{1} \tilde{\mathbf{s}}_{i} \dot{\mathbf{J}}_{w i}-e_{1} \mathbf{s}_{i} \cdot\left(\mathbf{J}_{w i} \dot{\mathbf{X}}\right)^{T} \mathbf{J}_{w i}, \\
\dot{\mathbf{v}}_{2 i}= & \ddot{d}_{i} \cdot \mathbf{s}_{i}+2 \dot{d}_{i}\left(\omega_{i} \times \mathbf{s}_{i}\right)+\left(d_{i}-e_{2}\right) \dot{\omega}_{i} \times \mathbf{s}_{i} \\
& +\left(d_{i}-e_{2}\right) \omega_{i} \times\left(\omega_{i} \times \mathbf{s}_{i}\right)=\mathbf{J}_{v 2 i} \ddot{\mathbf{X}}+\dot{\mathbf{J}}_{v 2 i} \dot{\mathbf{X}}, \\
\dot{\mathbf{J}}_{v 2 i}= & \mathbf{s}_{i} \cdot \dot{\mathbf{J}}_{0 i}-2 \mathbf{J}_{0 i} \cdot \dot{\mathbf{X}} \cdot \tilde{\mathbf{s}}_{i} \cdot \mathbf{J}_{w i} \\
& +\left(e_{2}-d_{i}\right)\left[\tilde{\mathbf{s}}_{i} \cdot \dot{\mathbf{J}}_{w i}+\left[\left(\mathbf{J}_{w i} \dot{\mathbf{X}}\right) \times\right] \tilde{\mathbf{s}} \cdot \dot{\mathbf{J}}_{w i}\right] .
\end{aligned}
$$

The detailed kinematic analysis has been developed indepth in our previous work [31], so herein, we do not repeat it.

\section{Dynamic Analysis}

The inverse dynamic problem of the parallel mechanism is to determine the required driving force under the requirement to generate a prescribed motion trajectory. For the 2RPU2SPR parallel mechanism, the dynamic model in the task space can be deduced by applying the principle of virtual work and d'Alembert's formulation stated as follows [32, 33]:

$$
\mathbf{F}_{X}=\mathbf{M}(\mathbf{X}) \ddot{\mathbf{X}}+\mathbf{C}(\dot{\mathbf{X}}, \mathbf{X}) \dot{\mathbf{X}}+\mathbf{N}(\mathbf{X}),
$$

where

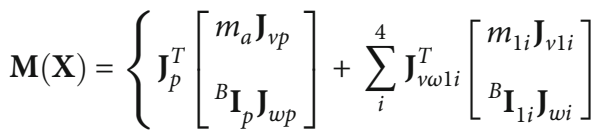

$$
\begin{aligned}
& \left.+\sum_{i}^{4} \mathbf{J}_{v \omega 2 i}^{T}\left[\begin{array}{l}
m_{2 i} \mathbf{J}_{v 2 i} \\
{ }^{B} \mathbf{I}_{2 i} \mathbf{J}_{w i}
\end{array}\right]\right\},
\end{aligned}
$$

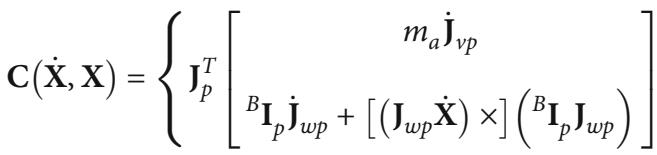

$$
\begin{aligned}
& +\sum_{i} \mathbf{J}_{v \omega 1 i}^{T}\left[\begin{array}{c}
m_{1 i} \dot{\mathbf{J}}_{v 1 i} \\
{ }^{B} \mathbf{I}_{1 i} \dot{\mathbf{J}}_{w i}+\left[\left(\mathbf{J}_{w i} \dot{\mathbf{X}}\right) \times\right]\left({ }^{B} \mathbf{I}_{1 i} \mathbf{J}_{w i}\right)
\end{array}\right] \\
& \left.+\sum_{i} \mathbf{J}_{v \omega 2 i}^{T}\left[\begin{array}{c}
m_{2 i} \dot{\mathbf{J}}_{v 2 i} \\
{ }^{B} \mathbf{I}_{2 i} \dot{\mathbf{J}}_{w i}+\left[\left(\mathbf{J}_{w i} \dot{\mathbf{X}}\right) \times\right]\left({ }^{B} \mathbf{I}_{2 i} \mathbf{J}_{w i}\right)
\end{array}\right]\right\}, \mathbf{N}(\mathbf{X}) \\
& =\left\{\mathbf{J}_{p}^{T}\left[\begin{array}{c}
m_{a} \mathbf{g} \\
0
\end{array}\right]+\sum_{i} \mathbf{J}_{v w 1}^{T}\left[\begin{array}{c}
m_{1 i} \mathbf{g} \\
0
\end{array}\right]\right. \\
& \left.+\sum_{i} \mathbf{J}_{v w 2}^{T}\left[\begin{array}{c}
m_{2 i} \mathbf{g} \\
0
\end{array}\right]\right\}-\mathbf{J}_{p}^{T}\left[\begin{array}{c}
\mathbf{f}_{e} \\
\mathbf{n}_{e}
\end{array}\right],
\end{aligned}
$$

where $\mathbf{M}(\mathbf{q})$ is the positive definite inertia matrix, $\mathbf{C}(\mathbf{q})$ is the nonlinear matrix including the centrifugal and the Coriolis terms, and $\mathbf{N}(\mathbf{X})$ is the sum of gravitational $f$ and external generalized force terms.

To facilitate force control, it is usually necessary to transform the dynamic formulation in the task operational space to that in the joint space.

In the static equilibrium state, the linear mapping relationship between the actuated joint force and corresponding task operation space force can be expressed as

$$
\mathbf{F}_{X}=\mathbf{J}_{0}^{T} \tau
$$

Combining (23) and (25) leads to

$$
\mathbf{J}_{0}^{T} \tau=\mathbf{M}(\mathbf{X})\left(\mathbf{J}_{0}{ }^{+} \ddot{\mathbf{q}}+\dot{\mathbf{J}}_{0}^{+} \dot{\mathbf{q}}\right)+C(\dot{\mathbf{X}}, \mathbf{X}) \mathbf{J}_{0}{ }^{+} \dot{\mathbf{q}}+\mathbf{N}(\mathbf{X}) .
$$

Then, the dynamic formulation in the joint space can be expressed with the deduced kinematic relations.

$$
\tau=\mathbf{M}(\mathbf{q}) \ddot{\mathbf{q}}+C(\dot{\mathbf{q}}, \mathbf{q}) \dot{\mathbf{q}}+\mathbf{N}(\mathbf{q}),
$$

where

$$
\begin{aligned}
\mathbf{M}(\mathbf{q}) & =\left(\mathbf{J}_{0}{ }^{T}\right)^{+} \mathbf{M}(\mathbf{X}) \mathbf{J}_{0}{ }^{+}, \\
\mathbf{C}(\dot{\mathbf{q}}, \mathbf{q}) & =\left(\mathbf{J}_{0}{ }^{T}\right)^{+}\left[\mathbf{C}(\mathbf{X})-\mathbf{M}(\mathbf{X}) \mathbf{J}_{0}{ }^{+} \mathbf{J}_{0}\right] \mathbf{J}_{0}{ }^{+}, \\
\mathbf{N}(\mathbf{q}) & =\left[\mathbf{J}_{0}{ }^{T}\right]^{+} \mathbf{N}(\mathbf{X}) .
\end{aligned}
$$


In addition, the dynamic model has several properties that can be exploited to facilitate dynamic controller designs; for example:

Property 1. The inertia matrix $\mathbf{M}(\mathbf{q})$ is symmetric and positive definite for any $\mathbf{q} \in R^{n}$.

Proof.

$$
\begin{aligned}
\mathbf{M}(\mathbf{q})^{T} & =\left[\left(\mathbf{J}_{0}^{+}\right)^{T} \mathbf{M}(\mathbf{X}) \mathbf{J}_{0}{ }^{+}\right]^{T} \\
& =\left(\mathbf{J}_{0}{ }^{+}\right)^{\mathrm{T}} \mathbf{M}(\mathbf{X}) \mathbf{J}_{0}{ }^{+}=\mathbf{M}(\mathbf{q}) .
\end{aligned}
$$

Property 2. $\dot{\mathbf{M}}(\mathbf{q})-2 \mathbf{C}(\dot{\mathbf{q}}, \mathbf{q})$ is skew symmetric for any $\mathbf{X}$, and the condition should be satisfied

$$
\mathbf{X}^{T}[\dot{\mathbf{M}}(\mathbf{q})-2 \mathbf{C}(\dot{\mathbf{q}}, \mathbf{q})] \mathbf{X}=0
$$

Proof.

$$
\begin{aligned}
\dot{\mathbf{M}}(\mathbf{q})-2 \mathbf{C}(\dot{\mathbf{q}}, \mathbf{q})= & \left(\dot{\mathbf{J}}_{0}^{+}\right)^{T} \mathbf{M}(\mathbf{X}) \mathbf{J}_{0}{ }^{+}+\left(\mathbf{J}_{0}{ }^{+}\right)^{T} \dot{\mathbf{M}}(\mathbf{X}) \mathbf{J}_{0}{ }^{+} \\
& +\left(\mathbf{J}_{0}^{+}\right)^{T} \mathbf{M}(\mathbf{X}) \dot{\mathbf{J}}_{0}^{+} \\
& -2\left(\mathbf{J}_{0}^{+}\right)^{T}\left[\mathbf{C}(\mathbf{X})-\mathbf{M}(\mathbf{X}) \mathbf{J}_{0}{ }^{+} \dot{\mathbf{J}}_{0}\right] \mathbf{J}_{0}{ }^{+} \\
= & \left(\mathbf{J}_{0}^{+}\right)^{T}[\dot{\mathbf{M}}(\mathbf{X})-2 \mathbf{C}(\mathbf{X})] \mathbf{J}_{0}{ }^{+} \\
& +\left(\dot{\mathbf{J}}_{0}^{+}\right)^{T} \mathbf{M}(\mathbf{X}) \mathbf{J}_{0}{ }^{+}-\left(\mathbf{J}_{0}{ }^{+}\right)^{T} \mathbf{M}(\mathbf{X}) \dot{\mathbf{J}}_{0}{ }^{+}
\end{aligned}
$$

due to

$$
\begin{aligned}
& \left(\dot{\mathbf{J}}_{0}^{+}\right)^{T} \mathbf{M}(\mathbf{X}) \mathbf{J}_{0}{ }^{+}-\left(\mathbf{J}_{0}{ }^{+}\right)^{T} \mathbf{M}(\mathbf{X}) \dot{\mathbf{J}}_{0}^{+} \\
& \quad+\left[\left(\dot{\mathbf{J}}_{0}^{+}\right)^{T} \mathbf{M}(\mathbf{X}) \mathbf{J}_{0}{ }^{+}-\left(\mathbf{J}_{0}^{+}\right)^{T} \mathbf{M}(\mathbf{X}) \dot{\mathbf{J}}_{0}^{+}\right]^{T}=0,
\end{aligned}
$$

and $\dot{\mathbf{M}}(\mathbf{X})-2 \mathbf{C}(\mathbf{X})$ is skew symmetric matrix.

Hence, $\dot{\mathbf{M}}(\mathbf{q})-2 \mathbf{C}(\dot{\mathbf{q}}, \mathbf{q})$ can be proven that it is a skew symmetric matrix.

\section{Control Design}

With due consideration of the fact that the dynamic formulations of the parallel mechanisms possess the same abovementioned properties as those of serial robots, we can apply the control scheme utilized for serial robots to parallel mechanisms with redundant actuation [34-36]. In order to achieve a good performance of parallel mechanism in trajectory tracking problem, an effective control scheme based on the nonlinear control theory is required. In this paper, we proposed a force/position hybrid control scheme to improve the trajectory tracking performance. Details are as follows:

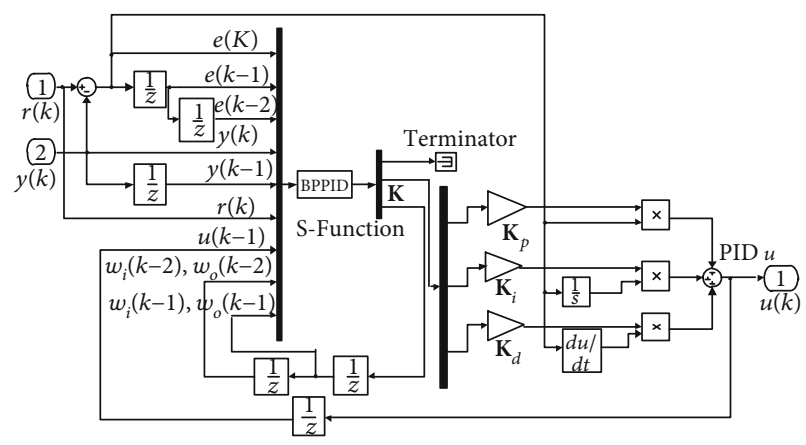

FIgURE 3: The diagram of the BP neural network PID controller.

5.1. Task Space PID (Proportional-Integral-Derivative) Position Control. Nonredundant limb (the $1^{\text {st }}, 2^{\text {nd }}$, and $3^{\text {rd }}$ limbs) adopts position control law combining inertia feedforward control and back propagation (BP) neural network (NN) PID control to guarantee the transient tracking precision performance. The control law for the proposed scheme can be expressed as follows:

$$
\begin{aligned}
\mathbf{u}_{p}= & \mathbf{M}(\mathbf{X}) \ddot{\mathbf{X}}_{d}+C(\dot{\mathbf{X}}, \mathbf{X}) \dot{\mathbf{X}}_{d}+\mathbf{N}(\mathbf{X}) \\
& +\mathbf{K}_{p} e+\mathbf{K}_{i} \int_{0}^{t} e \mathrm{~d} t+\mathbf{K}_{d} \dot{e},
\end{aligned}
$$

in which $\mathbf{X}_{d}$ is the desired trajectory in the task (operational) workspace; $\dot{\mathbf{X}}_{d}$ and $\ddot{\mathbf{X}}_{d}$ are the desired velocity and acceleration, respectively; and $\mathbf{K}_{p}, \mathbf{K}_{i}$, and $\mathbf{K}_{d}$ are positive definite suitable gain matrices.

Combining (31) and (41), and simplifying, we can get

$$
\begin{aligned}
\mathbf{M}(\mathbf{X})\left(\ddot{\mathbf{X}}_{d}-\ddot{\mathbf{X}}\right)+C(\dot{\mathbf{X}}, \mathbf{X})\left(\dot{\mathbf{X}}_{d}-\dot{\mathbf{X}}\right)+\mathbf{K}_{p} e \\
\quad+\mathbf{K}_{i} \int_{0}^{t} e \mathrm{~d} t+\mathbf{K}_{d} \dot{e}=0,
\end{aligned}
$$

namely,

$$
\mathbf{M}(\mathbf{X}) \ddot{\mathbf{e}}+C(\dot{\mathbf{X}}, \mathbf{X}) \dot{\mathbf{e}}+\mathbf{K}_{p} \mathbf{e}+\mathbf{K}_{i} \int_{0}^{t} \mathbf{e d} t+\mathbf{K}_{d} \dot{\mathbf{e}}=0,
$$

where $\mathbf{e}=\mathbf{X}_{d}-\mathbf{X}$ and $\dot{\mathbf{e}}=\dot{\mathbf{X}}_{d}-\dot{\mathbf{X}}$; if time is infinity, and the error $\mathbf{e}$ will evolve towards zero, then the derivative of the error $\dot{\mathbf{e}}$ will evolve towards zero as well.

Position control can guarantee the trajectory movement precision. The nonredundant limbs are controlled by the BP neural network PID controller. Figure 3 shows the block diagram of the $\mathrm{BP}$ neural network model which is employed in the intelligent gain tuning by online learning [37-41]. In this model, the NN has four input layers, eight hidden layers, and three output layers, and $w_{o}$ and $w_{i}$ are the weight factors of input layers and output layers that can be continuously updated by machine learning. The BP neural network PID controller is implemented by resorting to the "S-Function."

Here, the input of the control function is considered as the output of the $\mathrm{NN}$, which can be formulated as follows: 


$$
\left\{\begin{array}{l}
u(k)=u(k-1)+\mathbf{K}\left\{\mathbf{K}_{p}[e(k)-e(k-1)]+\mathbf{K}_{i} e(k)+\mathbf{K}_{d}[e(k)-2 e(k-1)+e(k-2)]\right\}, \\
e(k)=r(k)-y(k),
\end{array}\right.
$$

where $u(k)$ is the output of the controller in time $k$ and $e(k)$ is the control error between input $r(k)$ and output $y(k)$. It is worth noting that the variables $u(k), r(k)$, and $y(k)$ are all in the range between zero and one, so we do not have to normalize them. However, we should define an amplification gain factor $\mathbf{K}$ that can modify the magnitude of PID, and $\mathbf{K}_{p}, \mathbf{K}_{i}$, and $\mathbf{K}_{d}$ are the gain matrices of proportion, integration, and differentiation, respectively.

5.2. Joint Space P (Proportional) Force Control. Utilizing the force control as input function of the redundantly actuated limb (the $4^{\text {th }} \operatorname{limb}$ ), the proportion control law can be defined as follows:

$$
\mathbf{u}_{f}=\tau_{d}+\mathbf{K}_{v}\left(\tau_{d}-\tau\right)
$$

where $\tau_{d}$ is the desired driving force, $\tau$ is the actual joint force, and $\mathbf{K}_{V}$ is the positive gain matrices.

Force control can guarantee the distribution of the driving forces for preventing the damage of unexpected dash. Because the forces in joint space can be more easily measured than in the task space, so we select the dynamic control in joint space.

Up to now, the whole hybrid control law applied to the parallel mechanism with redundant actuation can hence be described as

$$
\begin{aligned}
\Gamma= & \mathbf{S}_{r m} \times\left[\mathbf{J}_{0}^{+}\right]^{T} u_{p}+\mathbf{S}_{r f} \times \mathbf{u}_{f} \\
= & \mathbf{S}_{r m} \times\left[\mathbf{J}_{0}^{+}\right]^{T}\left[\mathbf{M}(\mathbf{X}) \ddot{\mathbf{X}}_{d}+\mathbf{C}(\dot{\mathbf{X}}, \mathbf{X}) \dot{\mathbf{X}}_{d}+\mathbf{N}(\mathbf{X})\right. \\
& \left.+\mathbf{K}_{p} e+\mathbf{K}_{i} \int_{0}^{t} e \mathrm{~d} t+\mathbf{K}_{d} \dot{e}\right]+\mathbf{S}_{r f} \times\left[\tau_{d}+\mathbf{K}_{v}\left(\tau_{d}-\tau\right)\right],
\end{aligned}
$$

where

$$
\begin{aligned}
& \mathbf{S}_{r m}=\left[\begin{array}{lll}
1 & 0 & 0 \\
0 & 1 & 0 \\
0 & 0 & 1 \\
0 & 0 & 0
\end{array}\right], \\
& \mathbf{S}_{r f}=\left[\begin{array}{l}
0 \\
0 \\
0 \\
1
\end{array}\right] .
\end{aligned}
$$

Therefore, $\mathbf{S}_{r m}$ determines the nonredundantly actuated joint to be controlled with position control, while $\mathbf{S}_{r f}$ determines the redundant actuated joint to be controlled with force/torque control. The integrated control scheme can guarantee the stability at high speed and high acceleration engineering application.

In order to validate the stability of the control law, the following candidate Lyapunov function is proposed:

$$
V=\frac{1}{2} \dot{\mathbf{e}}^{T} \mathbf{M}(\mathbf{X}) \dot{\mathbf{e}}+\frac{1}{2} \mathbf{e}^{T} \mathbf{K}_{p} \mathbf{e}
$$

Taking into consideration positive defined matrices of $\mathbf{M}(\mathbf{X})$ and $\mathbf{K}_{p}$, leads to

$$
V \geq 0
$$

Differentiating $V$ with regard to time, meanwhile, noting (31) and (38), yields

$$
\begin{aligned}
\dot{V} & =\dot{\mathbf{e}}^{T} \mathbf{M}(\mathbf{X}) \ddot{\mathbf{e}}+\frac{1}{2} \dot{\mathbf{e}}^{T} \dot{\mathbf{M}}(\mathbf{X}) \dot{\mathbf{e}}+\dot{\mathbf{e}}^{T} \mathbf{K}_{p} \mathbf{e} \\
& =\dot{\mathbf{e}}^{T}\left(\mathbf{M}(\mathbf{X}) \ddot{\mathbf{e}}+\mathbf{C}(\dot{\mathbf{X}}, \mathbf{X}) \dot{\mathbf{e}}+\mathbf{K}_{p} \mathbf{e}\right) \\
& =-\dot{\mathbf{e}}^{T} K_{d} \dot{\mathbf{e}}-\dot{\mathbf{e}}^{T} K_{i} \int_{0}^{t} \mathbf{e d} t \leq-\dot{\mathbf{e}}^{T} K_{d} \dot{\mathbf{e}} \leq 0 .
\end{aligned}
$$

The parallel mechanism system will be stable, and $\mathbf{e} \rightarrow 0$ and $\dot{\mathbf{e}} \rightarrow 0$, as the time is infinity.

In order to demonstrate that the force/position hybrid control scheme combining the BP neural network PID control can effectively improve the trajectory tracking accuracy performance, the traditional PID also has been formulated for comparison analysis, as shown in Figure 4. The parallel manipulator is modeled by SolidWorks software, then is imported to SimMechanics through the SimMechanics Link plug-in; finally, Simulink module is established.

The desired trajectory parameter $\mathbf{X}_{d}$ can be obtained once the trajectory function was given. Herein, we present two methods to require the actual operational task space parameter $\mathbf{X}$. One is forward kinematics with the black line depicted in Figure 4. In the forward kinematics, the position of the actuated joints is known, then the position and orientations of the moving platform can be obtained by adopting the back propagation (BP) neural network optimization method or Newton-Raphson iteration, which converges rather quickly when the initial values are close to the desired solution $[42,43]$. However, the solving 


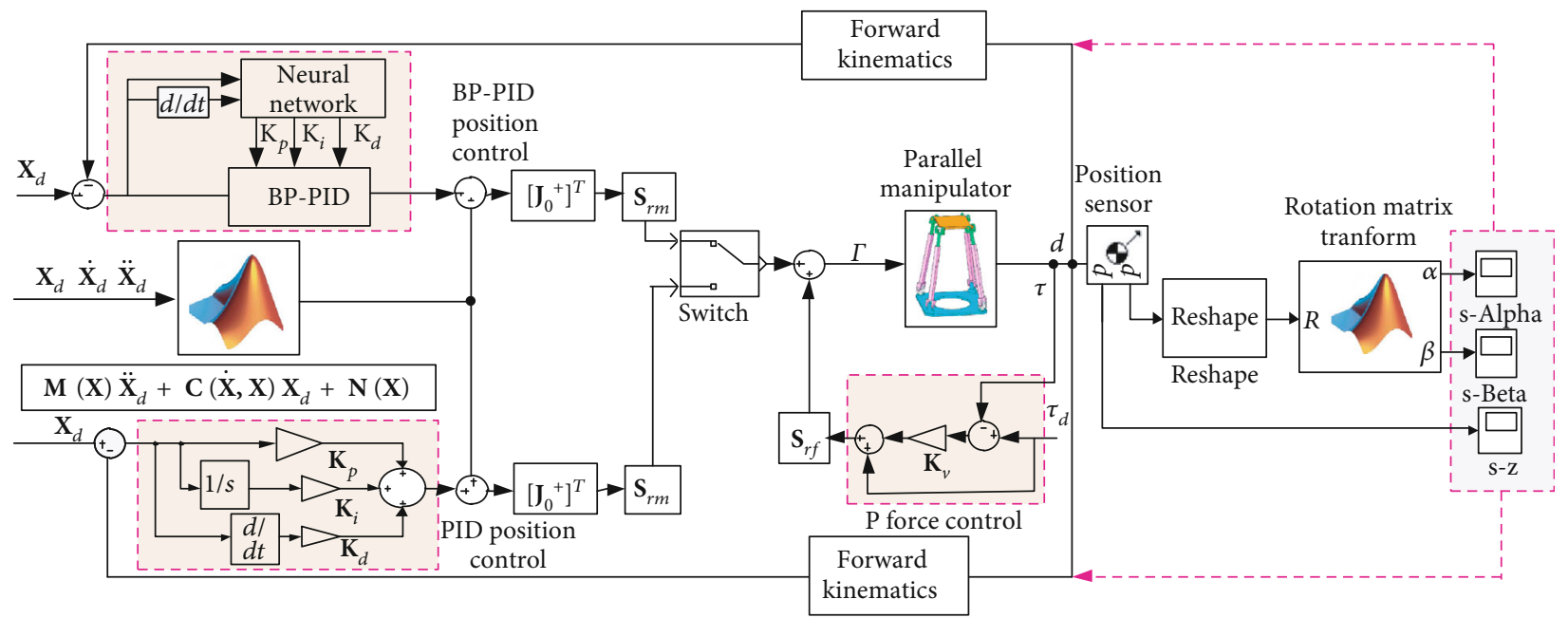

FIGURE 4: Force/position hybrid control scheme diagram of parallel mechanism with redundant actuation.

procedure for forward kinematics is very complicated and the computational efficiency is very low. Another efficient method to require the position and orientations of the moving platform is exactly what we want to introduce in this paper. We know that the position $z$. The rotation matrix $\mathbf{R}$ defining the orientation of the moving platform with respect to the fixed coordinate system can be obtained by position sensors. We can obtain the rotational Euler angles by interfacing with MATLAB in terms of the relation equation (2), just as shown in Figure 4, the dotted line within magenta. It is computationally simple and it does not require measurement of the end-effector velocity and acceleration as well. In fact, during the real prototype test, we can measure the plane change of the detected moving platform through the binocular version sensors; that is to say, we can obtain the rotation matrix. By resorting to the relationship equation (2), we can directly obtain the orientation angles $\alpha$ and $\beta$ in the workspace. The advantage of the proposed simplified method is that it can efficiently avoid the complex forward kinematics.

\section{Simulation Results}

In order to validate the performance of the proposed control method, first of all, the control scheme for the parallel mechanism simulation examples has been actually implemented within a modular MATLAB/Simulink programming environment [44]. The geometric parameters used for the parallel mechanism are listed as follows: the radius of the fixed platform is $r a=0.2 \mathrm{~m}$, the radius of the moving platform is $r b=0.339 \mathrm{~m}$, the mass of the moving platform is $m_{a}=12.75 \mathrm{~kg}$, the mass of the lower sublimb is $m_{1 i}=$ $0.95 \mathrm{~kg}$, the mass of the upper sublimb is $m_{2 i}=1.1 \mathrm{~kg}$, the distance between the centroid of the lower sublimb and the kinematic joint $B_{i}$ is $e_{1}=0.23 \mathrm{~m}$, the distance between the centroid of the upper sublimb and the joint $A_{i}$ is $e_{2}=$ $0.22 \mathrm{~m}$, the gravity acceleration is $\mathbf{g}=\left[\begin{array}{lll}0 & 0 & -9.807\end{array}\right]^{T}$, and the inertia matrix about the center of mass of the $i$-th limb can be determined by the SolidWorks software as follows (unit: $\mathrm{kg} \times \mathrm{m}^{2}$ ):

$$
\begin{aligned}
{ }^{B} \mathbf{I}_{p} & =\left[\begin{array}{ccc}
0.77 & 0 & 0 \\
0 & 1.07 & 0 \\
0 & 0 & 1.83
\end{array}\right] \times 10^{-1}, \\
{ }^{i} \mathbf{I}_{1 i} & =\left[\begin{array}{ccc}
2.88 & 0 & 0 \\
0 & 2.89 & 0 \\
0 & 0 & 0.05
\end{array}\right] \times 10^{-2}, \\
{ }^{i} \mathbf{I}_{2 i} & =\left[\begin{array}{ccc}
2.65 & 0 & 0 \\
0 & 2.67 & 0 \\
0 & 0 & 0.03
\end{array}\right] \times 10^{-2}
\end{aligned}
$$

A prescribed trajectory in spatial space is tracked using the proposed control scheme. Specifically, the desired trajectory function of the moving platform in the workspace is defined as follows:

$$
\left\{\begin{array}{l}
\alpha(t)=\frac{\pi}{6} \sin (2 t), \\
\beta(t)=\frac{\pi}{6} \cos (2 t), \\
z=0.7+0.02 \sin (2 t) .
\end{array}\right.
$$

The following values can be determined with tuning in the present traditional PID position control and P force control simulations:

$$
\begin{aligned}
& \mathbf{K}_{p}=\operatorname{diag}[1100,1100,1100,1100], \\
& \mathbf{K}_{i}=[20,20,20,20], \\
& \mathbf{K}_{d}=[20,20,20,20], \\
& \mathbf{K}_{v}=\operatorname{diag}[120,120,120,120] .
\end{aligned}
$$




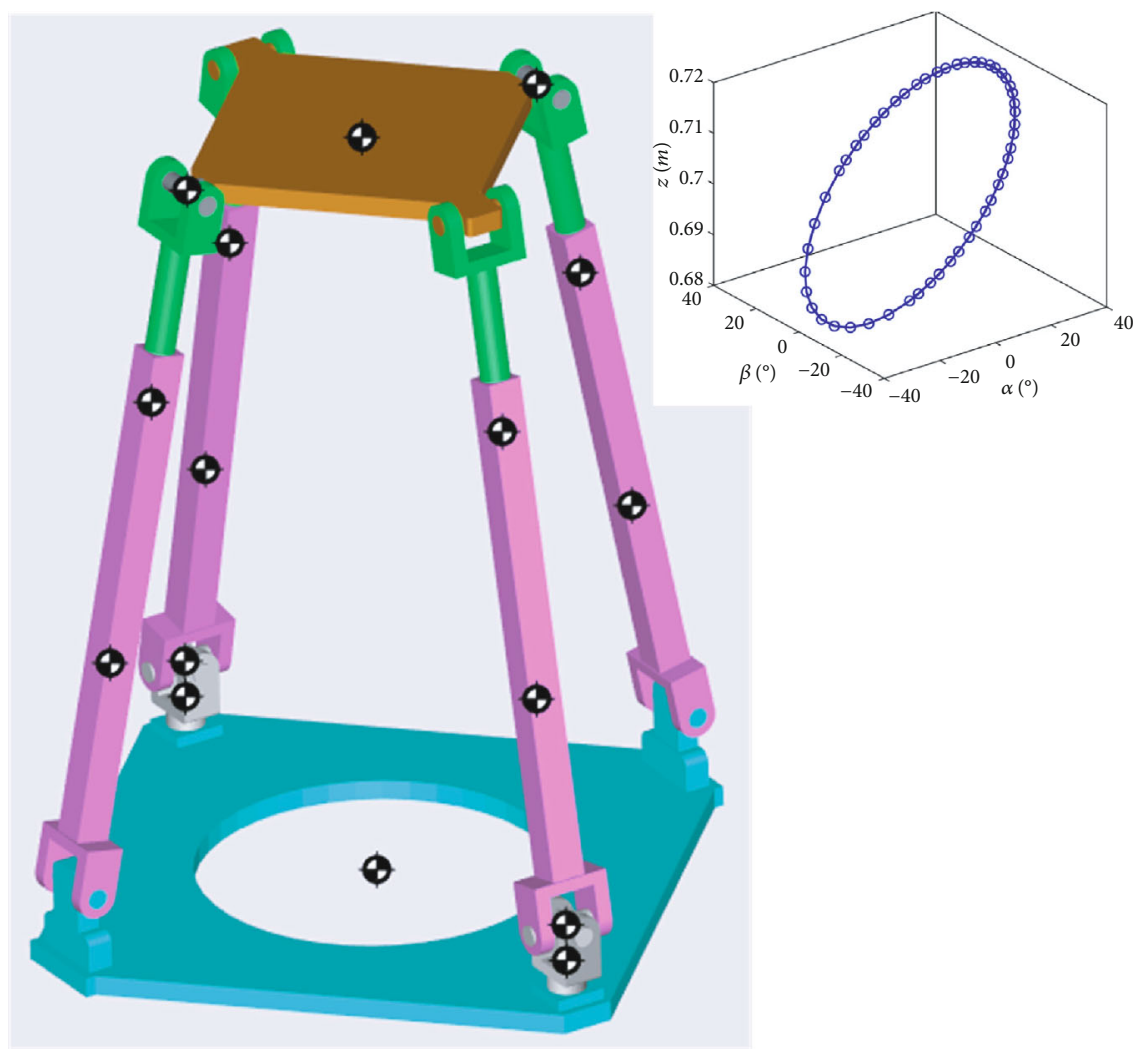

FIGURE 5: Parallel mechanism model in Simulink and desired trajectory.

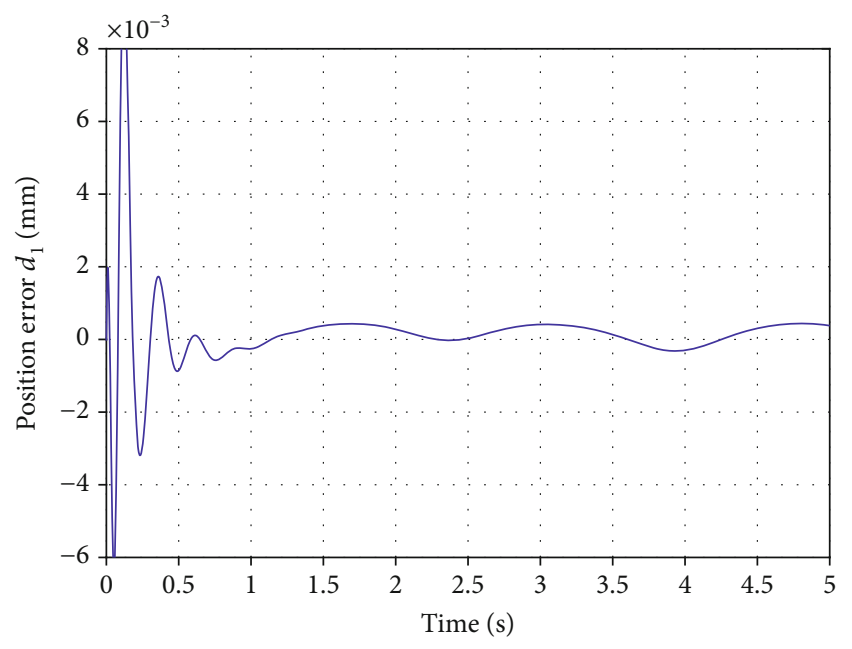

Figure 6: The position error of the first actuated joint $d_{1}$.

In BP neural network PID simulations, the weight factors are initialized from zero, while BP neural network PID $\left(\mathbf{K}_{p}, \mathbf{K}_{i}\right.$, and $\left.\mathbf{K}_{d}\right)$ control gain matrices are set to one, the amplification gain matrix $\mathbf{K}$ is set as $\operatorname{diag}[8700,300,70]$, and $\mathbf{K}_{v}$ is the same as the abovementioned. In the simulation process, the fixed simulation step (fundamental sample time) is set to $1 \mathrm{~ms}$, the model was simulated using the BogackiShampine method, and the simulation time is set to 5 seconds.

The Simulink simulation model and desired trajectory are depicted in Figure 5.

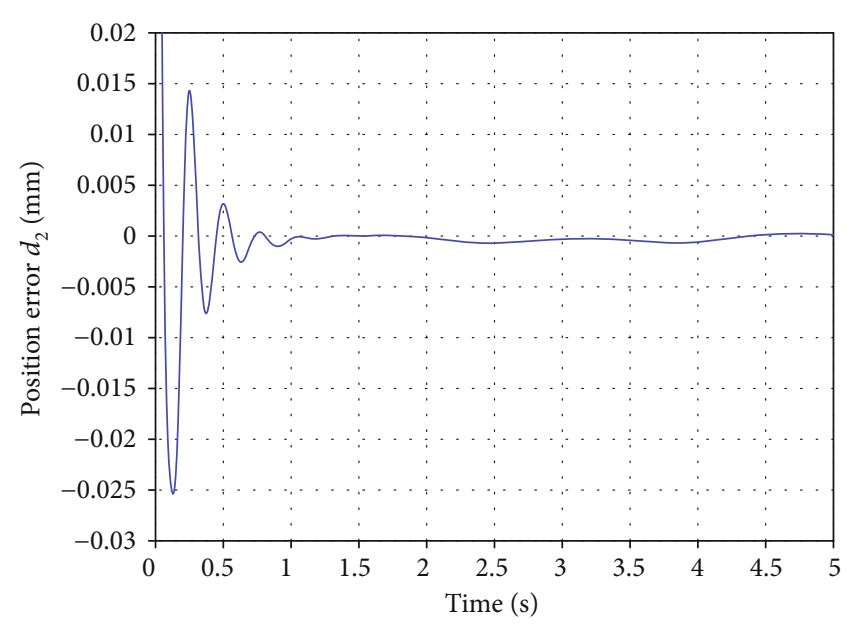

Figure 7: The position error of the second actuated joint $d_{2}$.

First of all, the proposed control scheme is applied to the parallel mechanism for the prescribed trajectory tracking control, and the position errors of the actuated joints are drawn in Figures 6-9. When the control system reaches a steady state, the errors will decrease gradually to zero, although the errors oscillated at the beginning or within some certain time.

From the figures above, we can see that the errors of the actuated joints are very small, and the variation mainly appears within one second. In the next four seconds, the errors almost approach zero, especially in Figures 7 and 9, while the errors in Figures 6 and 8 have small fluctuations 


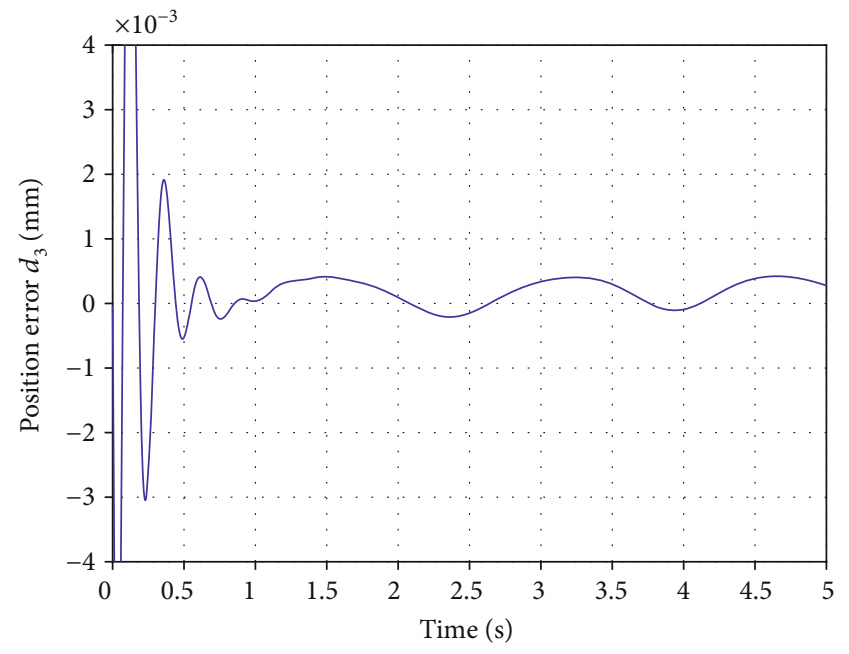

Figure 8: The position error of the third actuated joint $d_{3}$.

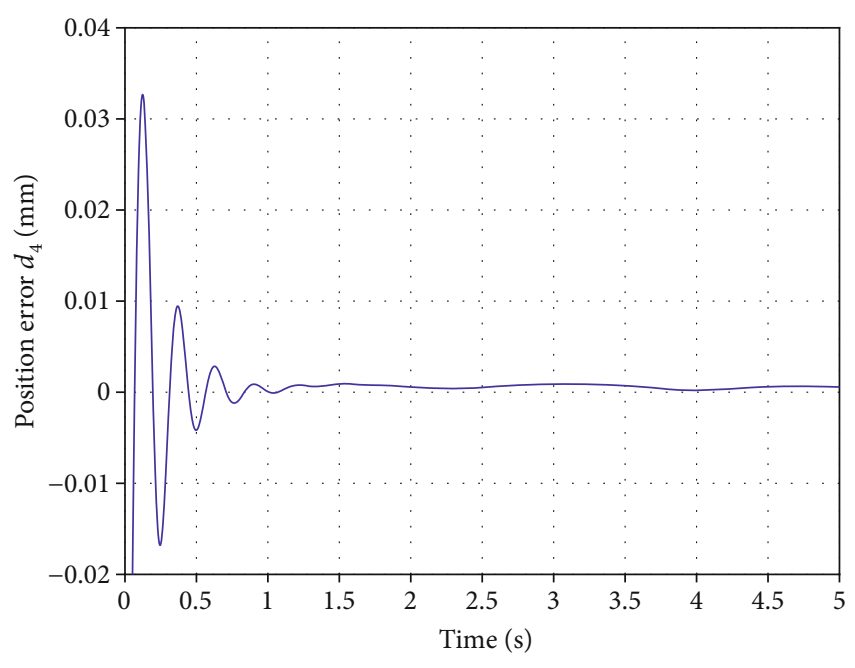

FIgURE 9: The position error of the fourth actuated joint $d_{4}$.

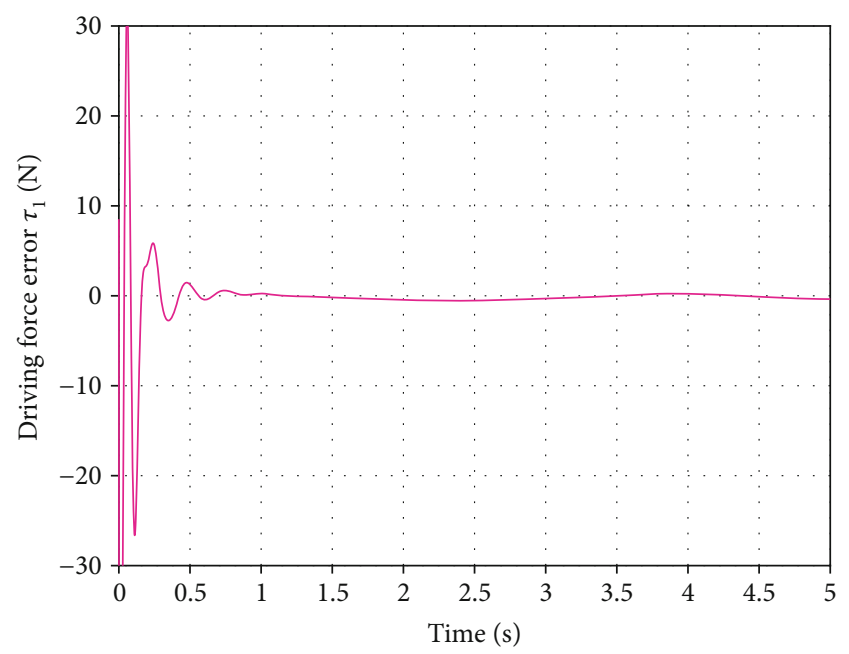

Figure 10: The driving force error of the first actuated joint.

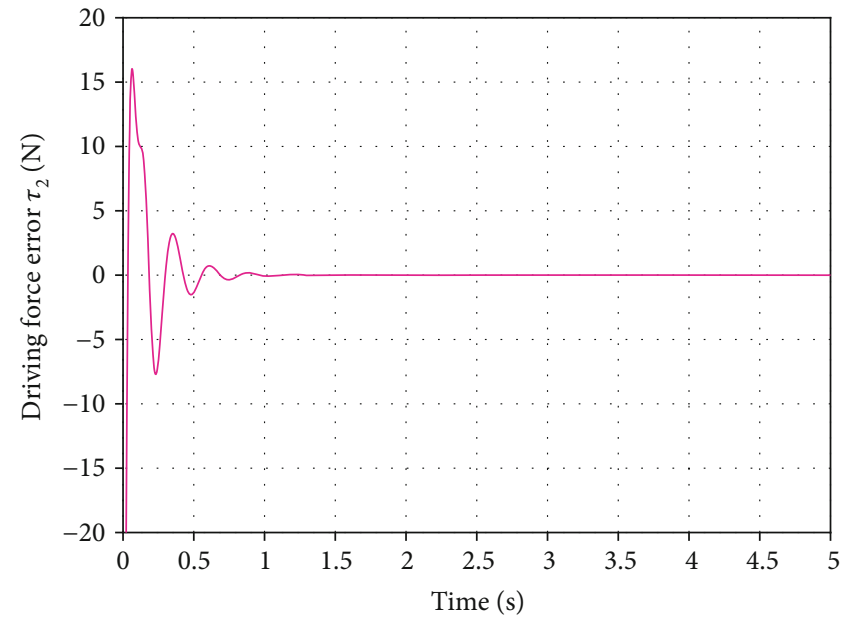

FIgURE 11: The driving force error of the second actuated joint.

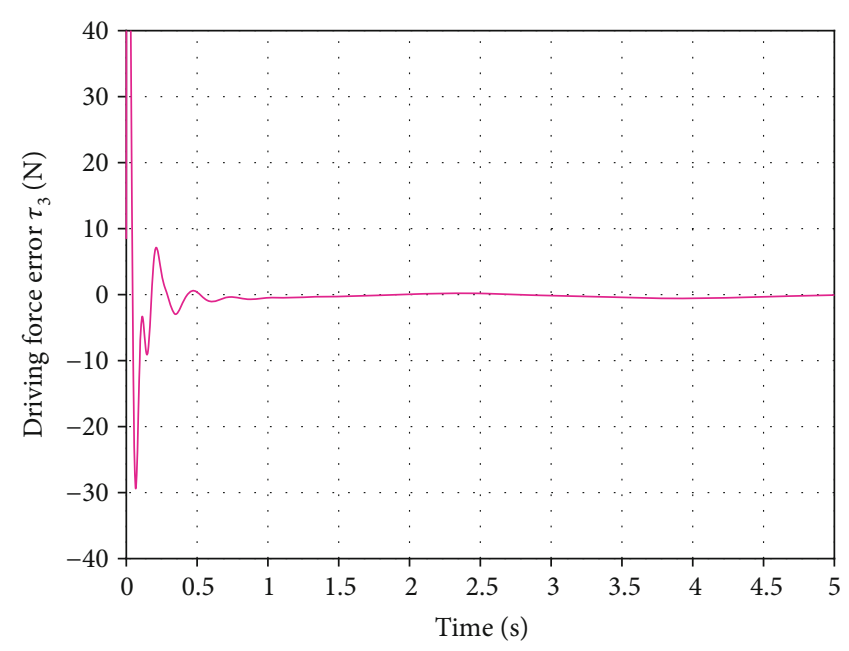

FIgURE 12: The driving force error of the third actuated joint.

that are approximately close to zero, which demonstrate that the designed controller has the advantage of quick response speed and excellent trajectory tracking results.

Similarly, the errors of driving forces are very important to validate the controller performance, and the simulation results including three nonredundant forces in the actuated joints are illustrated in Figures 10-12.

From the figures above, we can see that the errors are getting very fast, and they are close to zero after one second; however, the errors have a large fluctuation within one second. The unexpected dash may destroy the structural components, so we should improve significantly and pay attention to this problem in practical application.

The errors including actuated positions and actuated forces in the above figures tend to zero as the control system reaches a steady state. During the transient response of the control system, the values of the errors produced will be oscillating.

Figures 13 and 14 show the required movement position and driving force for each actuated joint of the parallel mechanism to follow the specified trajectory; herein, the 


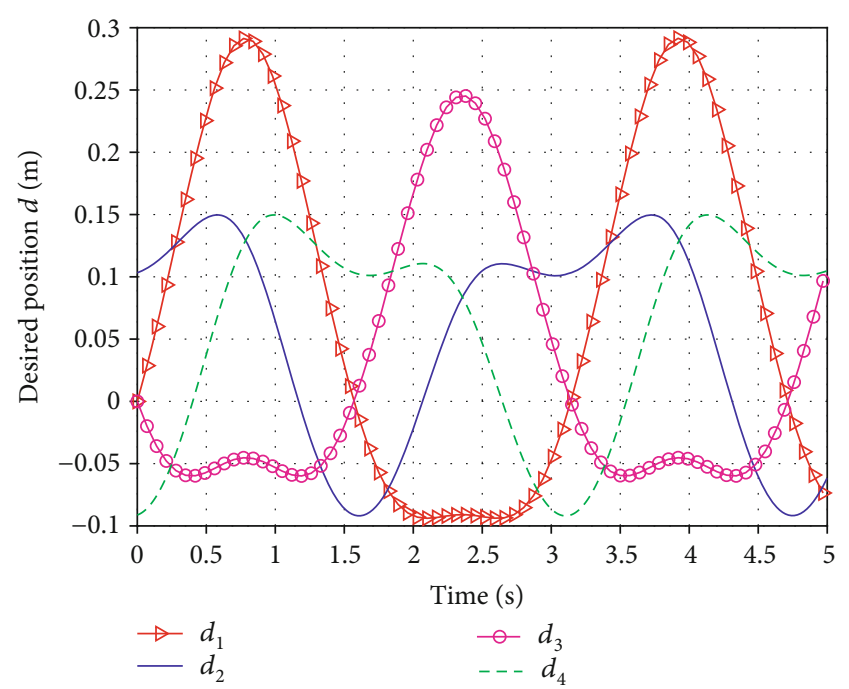

Figure 13: The desired position of the actuated joint $d$.

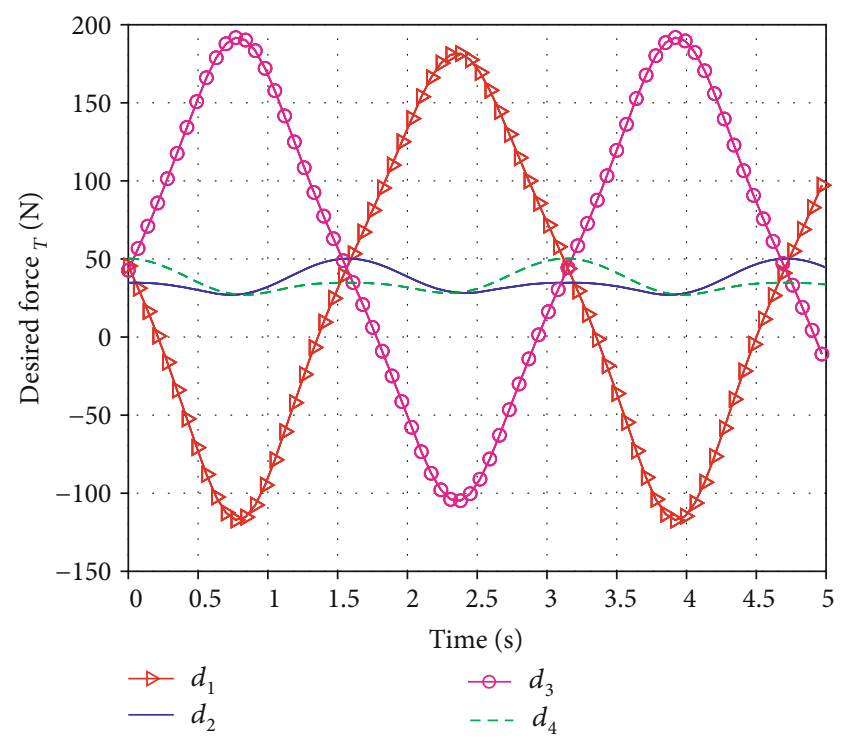

FIgURE 14: The desired driving force of the actuated joint.

force/position hybrid control scheme (the $1^{\text {st }} \operatorname{limb}, 2^{\text {nd }}$ limb, and $3^{\text {rd }}$ limb utilized position control, while the $4^{\text {th }}$ limb employed force control) is adopted.

In Figure 13, the changes of the actuated joint distances are stable and the amplitudes are approximately consistent. However, in Figure 14, the changes of the driving force are very apparent; what is more, the forces have a big difference between the $1^{\text {st }}$ driving force and the $4^{\text {th }}$ one. The comparison analysis demonstrates that the redundantly actuated parallel mechanism has the advantage of optimizing the driving force configuration over the movement process. But most importantly, we should consider the force control in trajectory tracking process, and it is very significant for the parallel mechanism with redundant actuation. In addition, Figure 14 illustrates that the driving forces $\tau_{1}, \tau_{2}$, $\tau_{3}$, and $\tau_{4}$ are the function about time. The values of actuator forces can be applied to choose the ideal drive motor. Appar-

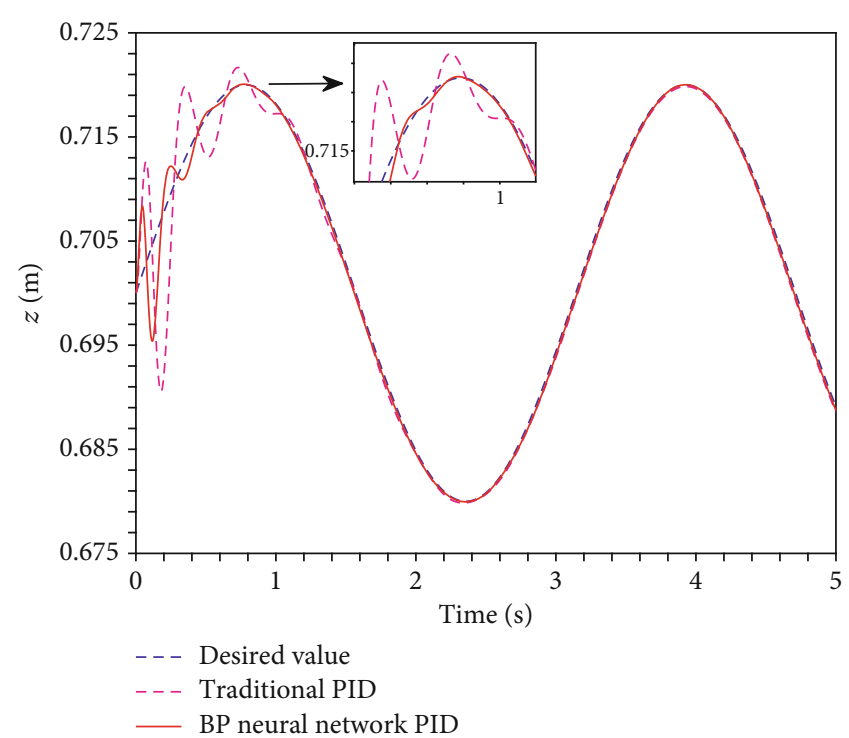

FIGURE 15: The trajectory tracking comparison in the $z$ direction.

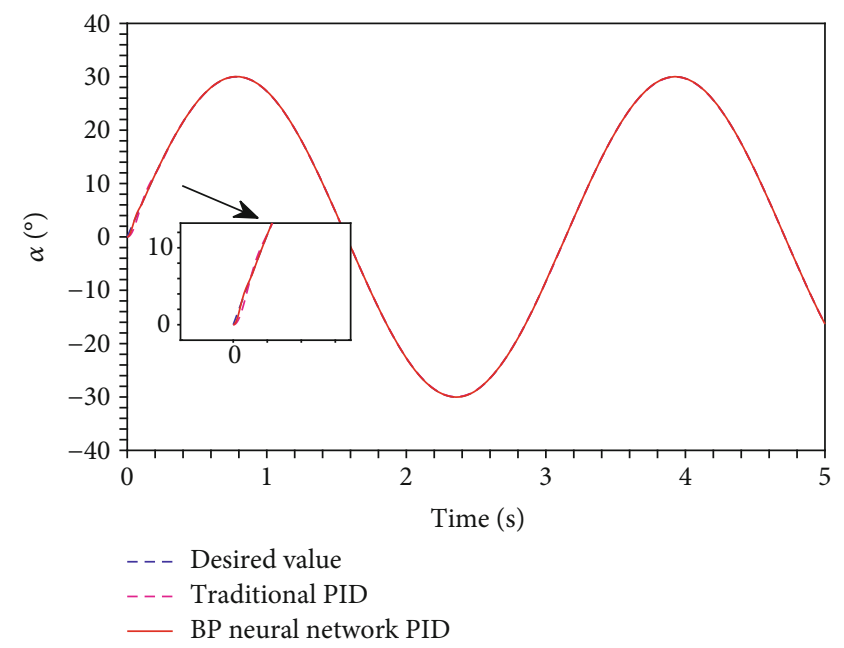

FIgURE 16: The trajectory tracking comparison about Euler angle $\alpha$.

ently, the parallel mechanism with redundant actuation can effectively decrease the magnitude of driving force and optimize the force configuration, which is a feature of the proposed new parallel mechanism.

In order to demonstrate the effectiveness of the proposed force/position hybrid control scheme for the execution of trajectory tracking, the two controllers, the BP neural network PID controller, and the traditional PID controller have been simulated simultaneously. As can readily be appreciated in Figures 15-17, the simulation results presented with both controllers are very similar. However, the traditional PID controller response speed is much worse, and we can see that the designed control scheme has a much better trajectory tracking performance than the traditional PID control method. The variation of Euler angle $\alpha$ is not apparent in the tracking process, as shown in Figure 16, but the designed control scheme has a quick response through the comparison analysis of Figures 15 and 17. The simulation 


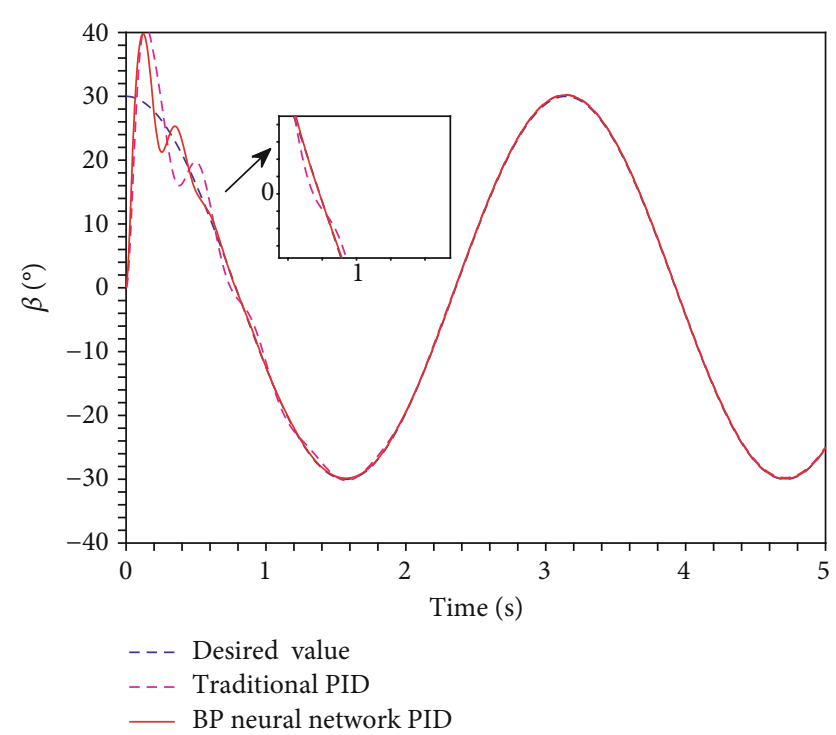

FIGURE 17: The trajectory tracking comparison about Euler angle $\beta$.

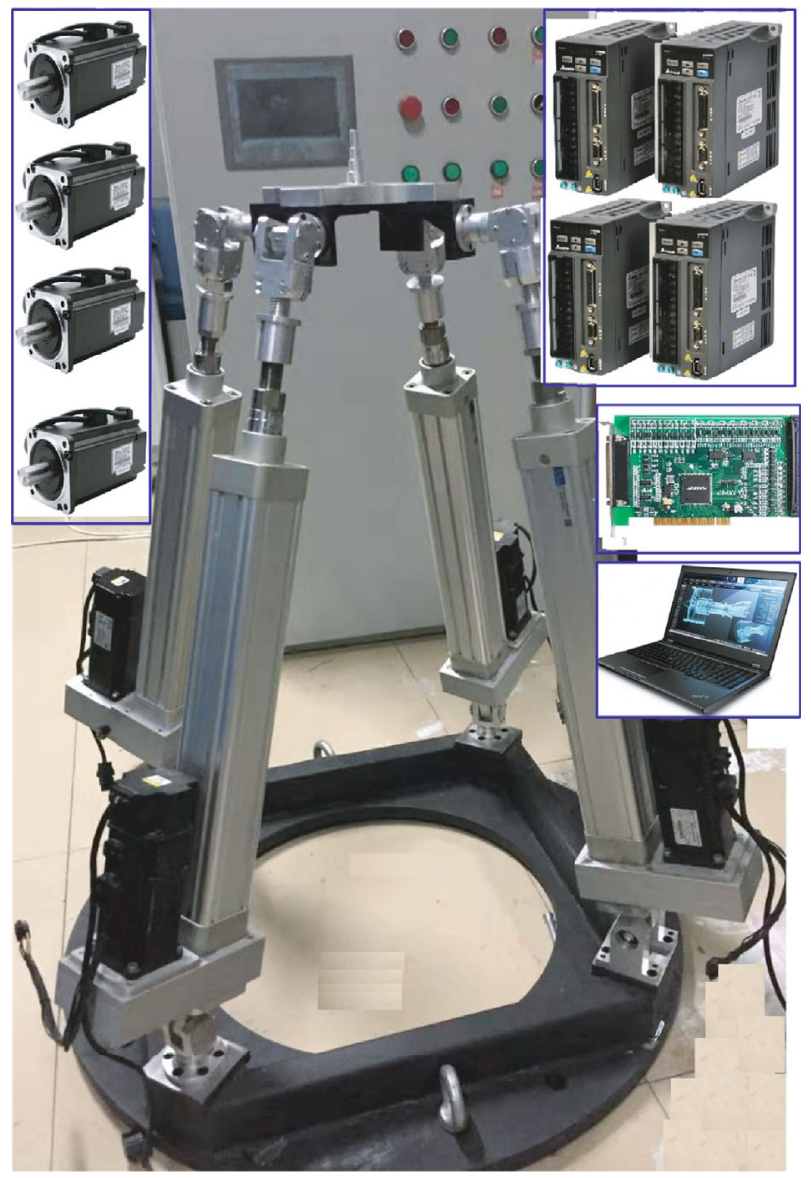

FIgURE 18: The experimental prototype of the parallel mechanism.

results verify the trajectory tracking performance of the proposed controller, which is available to control the parallel mechanism with redundant actuation for the most important application.
The kinematic and dynamic analyses established the theoretical foundation for intelligent control of the parallel mechanism. In the short run, we will concentrate on calibrating the errors by experiments. The experimental prototype is shown in Figure 18; the experimental system mainly consists of the parallel mechanism, four driver controllers, a multiaxis controller, and a master CPU controller. The real position and velocity of the four actuators will be transmitted to the computer for data monitoring and implementing control in real time.

\section{Conclusions}

In this paper, the trajectory tracking control scheme for a new 2RPU-2SPR parallel mechanism with redundant actuation is systematically investigated, and the following contributions can be obtained:

(1) The inverse kinematic analysis of the proposed parallel mechanism has been formulated, and the mapping relationship of velocity and acceleration between the joint space and task space are straightforwardly deduced including the inverse Jacobian matrix and the forward Jacobian matrix

(2) In terms of the principle of virtual work and the d'Alembert formulation, the inverse and forward dynamic standard formulation of the proposed parallel mechanism with redundant actuation is established and the optimal driving force value is obtained by utilizing the generalized Moore-Penrose inverse matrix

(3) On the basis of the kinematic and dynamic formulations, the force/position hybrid control by means of inertia feed-forward control and BP neural network PID control was implemented in MATLAB/Simulink. As an illustrative case, the simulation results verified the effectiveness of the force/position hybrid control law which can possess good trajectory tracking performance as compared with the traditional PID controller. Our future works will concentrate upon the effective intelligent controllers for versatile applications of high speed and high precision serialparallel hybrid kinematic machine tool

\section{Data Availability}

The data used to support the findings of this study are available from the corresponding authors upon request.

\section{Conflicts of Interest}

The authors declared no potential conflicts of interest with respect to the research, authorship, and/or publication of this article.

\section{Acknowledgments}

The authors would like to acknowledge the financial support of the Fundamental Research Funds for the Central 
Universities under Grant Nos. 2018JBZ007, 2018YJS136, and 2017YJS158; the China Scholarship Council (CSC) under Grant No. 201807090079; the Natural Sciences and Engineering Research Council of Canada (NSERC); and the York Research Chairs (YRC) program. Meanwhile, the author, Haiqiang Zhang, is grateful to the Advanced Robotics and Mechatronics Laboratory in York University and the science librarian John Dupuis.

\section{References}

[1] A. Allagheband, M. Mahmoodi, J. K. Mills, and B. Benhabib, "Comparative analysis of a redundant pentapod parallel kinematic machine," Journal of Mechanisms and Robotics, vol. 7, no. 3, article 034502, 2015.

[2] Q. Li, J. M. Hervé, and P. Huang, "Type synthesis of a special family of remote center-of-motion parallel manipulators with fixed linear actuators for minimally invasive surgery," Journal of Mechanisms and Robotics, vol. 9, no. 3, article 031012, 2017.

[3] H. Q. Zhang, H. R. Fang, and B. S. Jiang, "Motion-force transmissibility characteristic analysis of a redundantly actuated and overconstrained parallel machine," International Journal of Automation and Computing, vol. 16, no. 2, pp. 150-162, 2019.

[4] W. W. Shang, S. Cong, and F. R. Kong, "Identification of dynamic and friction parameters of a parallel manipulator with actuation redundancy," Mechatronics, vol. 20, no. 2, pp. 192-200, 2010.

[5] M. Diaz-Rodriguez, A. Valera, V. Mata, and M. Valles, "Model-based control of a 3-DOF parallel robot based on identified relevant parameters," IEEE/ASME Transactions on Mechatronics, vol. 18, no. 6, pp. 1737-1744, 2013.

[6] M. Heydarzadeh, N. Karbasizadeh, and M. T. Masouleh, "Experimental kinematic identification and position control of a 3-DOF decoupled parallel robot," Proceedings of the Institution of Mechanical Engineers, Part C: Journal of Mechanical Engineering Science, vol. 233, no. 5, pp. 1841-1855, 2018.

[7] G. Tao, C. Shang, and D. Meng, "Adaptive robust posture control of a 3-RPS pneumatic parallel platform with unknown deadzone," Mathematical Problems in Engineering, vol. 2016, Article ID 2034923, 16 pages, 2016.

[8] N. Kamaldin, S. L. Chen, C. S. Teo, W. Lin, and K. K. Tan, “A novel adaptive jerk control with application to large workspace tracking on a flexure-linked dual-drive gantry," IEEE Transaction on Industrial Electronics, vol. 66, no. 7, pp. 5353-5363, 2019.

[9] R. Hao, J. Z. Wang, J. Zhao, and S. Wang, "Robust tracking control of 6-DOF parallel electrical manipulator in joint-task space with fast friction estimation," IFAC Proceedings Volumes, vol. 47, no. 3, pp. 3857-3862, 2014.

[10] J.-L. Chang, H.-C. Ting, and Y.-P. Chen, "Robust discrete-time output tracking controller design for nonminimum phase systems," Journal of System Design and Dynamics, vol. 2, no. 4, pp. 950-961, 2008.

[11] B. Achili, B. Daachi, Y. Amirat, and A. Ali-cherif, "A robust adaptive control of a parallel robot," International Journal of Control, vol. 83, no. 10, pp. 2107-2119, 2010.

[12] A. Muller, "Redundant actuation of parallel manipulators," in Parallel Manipulators, Towards New Applications, H. Wu, Ed., vol. 4, pp. 87-108, IntechOpen, 2012.
[13] C. Gosselin and L. T. Schreiber, "Kinematically redundant spatial parallel mechanisms for singularity avoidance and large orientational workspace," IEEE Transactions on Robotics, vol. 32, no. 2, pp. 286-300, 2016.

[14] H. T. Liu, T. Huang, A. Kecskeméthy, D. G. Chetwynd, and Q. Li, "Force/motion transmissibility analyses of redundantly actuated and overconstrained parallel manipulators," Mechanism and Machine Theory, vol. 109, pp. 126-138, 2017.

[15] J. T. Yao, W. D. Gu, Z. Q. Feng, and Y. D. Xu, “Dynamic analysis and driving force optimization of a 5-DOF parallel manipulator with redundant actuation," Robotics and ComputerIntegrated Manufacturing, vol. 48, pp. 51-58, 2017.

[16] R. Boudreau, J. Léger, H. Tinaou, and A. Gallant, "Dynamic analysis and optimization of a kinematically redundant planar parallel manipulator," Transactions of the Canadian Society for Mechanical Engineering, vol. 42, no. 1, pp. 20-29, 2018.

[17] R. Boudreau and S. Nokleby, "Force optimization of kinematically-redundant planar parallel manipulators following a desired trajectory," Mechanism and Machine Theory, vol. 56, pp. 138-155, 2012.

[18] X. Y. Wang, S. Guo, H. B. Qu, and F. Q. Zhao, "Optimal allocation method of parallel mechanism and its application," Journal of Mechanical Engineering, vol. 55, no. 1, pp. 32-41, 2019.

[19] S. Wen, B. Zhang, P. Hao, H.-k. Lam, and H. Wang, "Fuzzy fractional order force control of 6PUS-UPU redundantly actuated parallel robot based on inner model position control structure," Engineering Application of Artificial Intelligence, vol. 65, pp. 200-211, 2017.

[20] S. Wen, J. Chen, G. Qin, Q. Zhu, and H. Wang, “An improved fuzzy model predictive control algorithm based on the force/position control structure of the five-degree of freedom redundant actuation parallel robot," International Journal of Advanced Robotic Systems, vol. 15, no. 5, 2018.

[21] X. F. Liu, J. T. Yao, and Y. S. Zhao, "Model-based synchronous control of redundantly actuated parallel manipulator with neural network," Transactions of the Chinese Society for Agricultural Machinery, vol. 49, no. 2, pp. 367-375, 2018.

[22] M. Luces, J. K. Mills, and B. Benhabib, "A review of redundant parallel kinematic mechanisms," Journal of Intelligent and Robotic Systems, vol. 86, no. 2, pp. 175-198, 2017.

[23] J. L. Flohic, F. Paccot, N. Bouton, and H. Chanal, "Application of hybrid force/position control on parallel machine for mechanical test," Mechatronics, vol. 49, pp. 168-176, 2018.

[24] J. Cazalilla, M. Vallés, V. Mata, M. Díaz-Rodríguez, and A. Valera, "Adaptive control of a 3-DOF parallel manipulator considering payload handling and relevant parameter models," Robotics and Computer-Integrated Manufacturing, vol. 30, no. 5, pp. 468-477, 2014.

[25] J. Cazalilla, M. Valles, and V. Mata, "Implementation of a 3DOF parallel robot adaptive motion controller," International Jounal of Mechanics and Control, vol. 15, no. 1, pp. 45-52, 2014.

[26] C. Urrea and H. Kern, "Design, simulation and comparison of controllers for a redundant robot," Case Studies in Mechanical System and Signal Processing, vol. 3, pp. 9-21, 2016.

[27] M. Callegari, M. C. Palpacelli, and M. Principi, "Dynamics modelling and control of the 3-RCC translational platform," Mechatronics, vol. 16, no. 10, pp. 589-605, 2006. 
[28] B. Siciliano, L. Sciavicco, L. Villani, and G. Oriolo, Robotics Modelling, Planning and Control, Springer, London, 2009.

[29] H. Zhang, H. Fang, Y. Fang, and B. Jiang, "Workspace analysis of a hybrid kinematic machine tool with high rotational applications," Mathematical Problems in Engineering, vol. 2018, Article ID 2607497, 12 pages, 2018.

[30] H. Zhang and H. Fang, "Stiffness characteristics analysis of a novel 3- DOF parallel kinematic machine tool," International Journal of Engineering and Technology, vol. 10, no. 4, pp. 346-354, 2018.

[31] H. Q. Zhang, H. R. Fang, B. S. Jiang, and S. G. Wang, "Dynamic performance evaluation of a redundantly actuated and over-constrained parallel manipulator," International Journal of Automation and Computing, vol. 16, no. 3, pp. 274-285, 2019.

[32] G. Cui, D. Zhang, H. Zhou, and Y. Zhang, "Operating dexterity optimization and analysis of a 3-DOF parallel manipulator for a tunnel segment assembly system," International Journal of Mechanics and Materials in Design, vol. 11, no. 3, pp. 277285, 2015.

[33] S. Lu and Y. Li, "Dynamic dexterity evaluation of a 3-DOF 3PUU parallel manipulator based on generalized inertia matrix," in 2015 IEEE International Conference on Robotics and Biomimetics (ROBIO), pp. 1506-1511, Zhuhai, China, 2015.

[34] T. S. Zhan and C. C. Kao, "Modified PSO method for robust control of 3RPS parallel manipulators," Mathematical Problems in Engineering, vol. 2010, Article ID 302430, 25 pages, 2010.

[35] W. W. Shang and S. Cong, "Dexterity and adaptive control of planar parallel manipulators with and without redundant actuation," Journal of Computational and Nonlinear Dynamics, vol. 10, no. 1, article 011002, 2015.

[36] C. Yang, Q. Huang, H. Jiang, O. O. Peter, and J. Han, "PD control with gravity compensation for hydraulic 6-DOF parallel manipulator," Mechanism and Machine Theory, vol. 45, no. 4, pp. 666-677, 2010.

[37] R. Hernández-Alvarado, L. García-Valdovinos, T. SalgadoJiménez, A. Gómez-Espinosa, and F. Fonseca-Navarro, "Neural network-based self-tuning PID control for underwater vehicles," Sensors, vol. 16, no. 9, article 1429, 2016.

[38] Y. A. Zhukov, E. B. Korotkov, A. V. Moroz, V. V. Zhukova, and A. M. Abramov, "Adaptive neural network control of hexapod for aerospace application," IOP Conference Series: Materials Science and Engineering, vol. 441, article 012001, 2018.

[39] A. Zribi, M. Chtourou, and M. Djemel, "A new PID neural network controller design for nonlinear processes," Journal of Circuits, Systems and Computers, vol. 27, no. 4, 2018.

[40] A. Zubizrreta, M. Larrea, E. Irigoyen, I. Cabanes, and E. Portillo, "Real time direct kinematic problem computation of the 3PRS robot using neural networks," Neurocomputing, vol. 271, pp. 104-114, 2018.

[41] M. Manderla, D. Schmitt, and U. Konigorsk, "Modelling, simulation and control of a redundant parallel robotic manipulator based on invariant manifolds," Mathematical and Computer Modeling of Dynamical Systems, vol. 16, no. 2, pp. 95-113, 2010.

[42] H. Zhang, H. Fang, D. Zhang, X. Luo, and Q. Zou, "Forward kinematics and workspace determination of a novel redundantly actuated parallel manipulator," International Journal of Aerospace Engineering, vol. 2019, Article ID 4769174, 14 pages, 2019.

[43] P. K. Jamwal, S. Q. Xie, Y. H. Tsoi, and K. C. Aw, "Forward kinematics modelling of a parallel ankle rehabilitation robot using modified fuzzy inference," Mechanism and Machine Theory, vol. 45, no. 11, pp. 1537-1554, 2010.

[44] F. Piltan, A. Taghizadegan, and N. Sulaiman, "Modeling and control of four degrees of freedom surgical robot manipulator using MATLAB/SIMULINK," International Journal of Hybrid Information Technology, vol. 8, no. 11, pp. 47-78, 2015. 


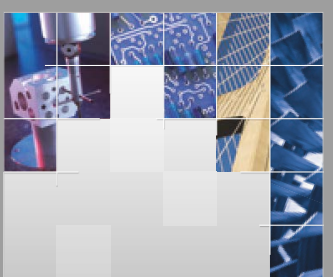

\section{Enfincering}
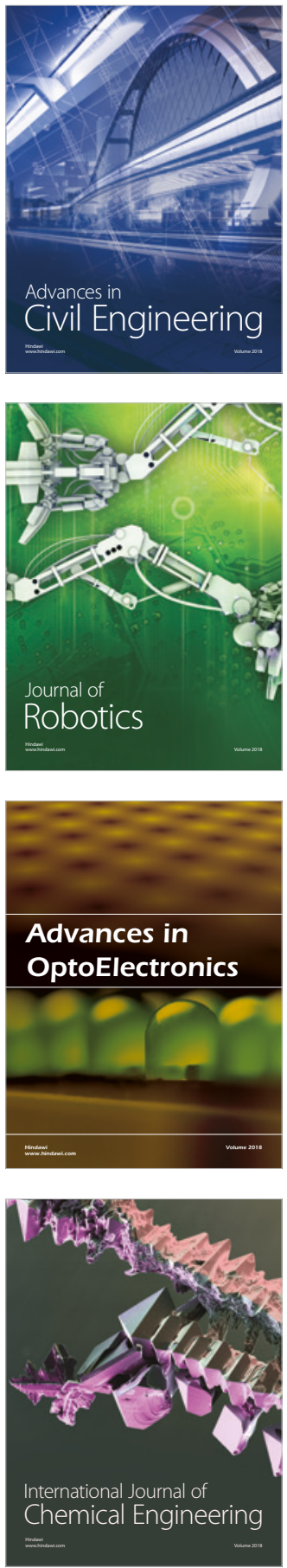

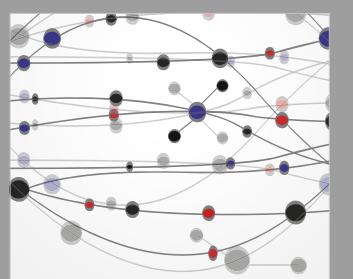

\section{Rotating \\ Machinery}

The Scientific World Journal

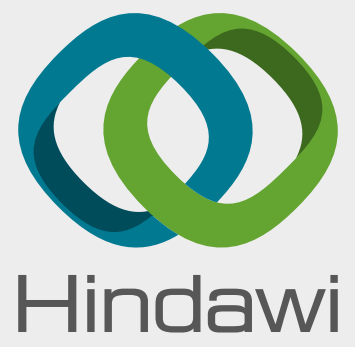

Submit your manuscripts at

www.hindawi.com
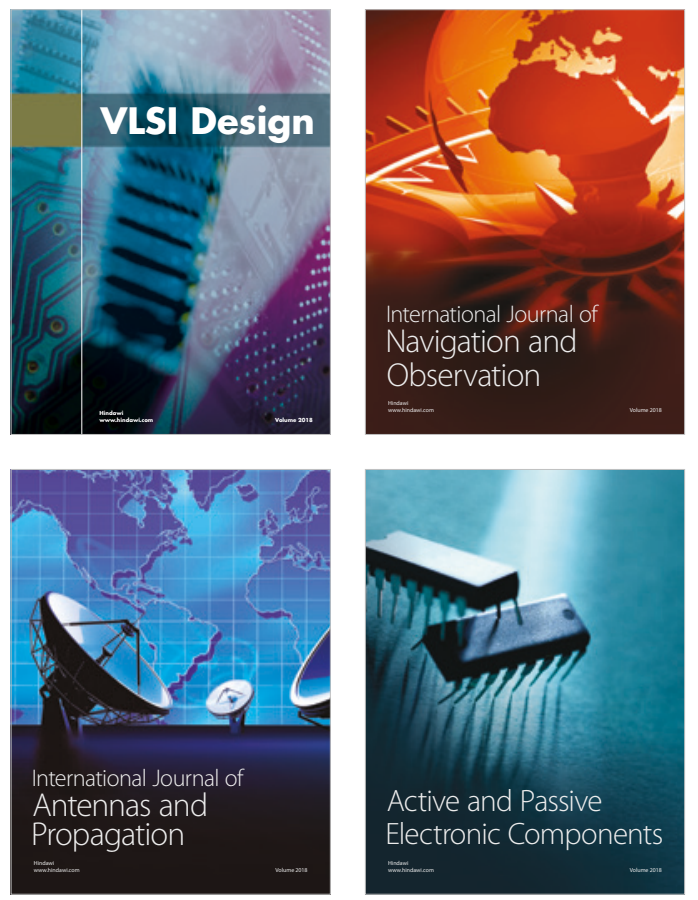
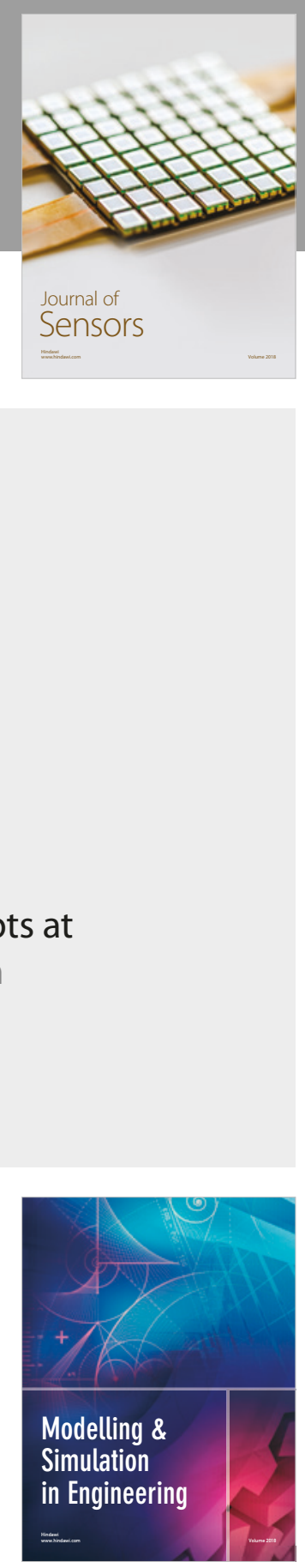

\section{Advances \\ Multimedia}
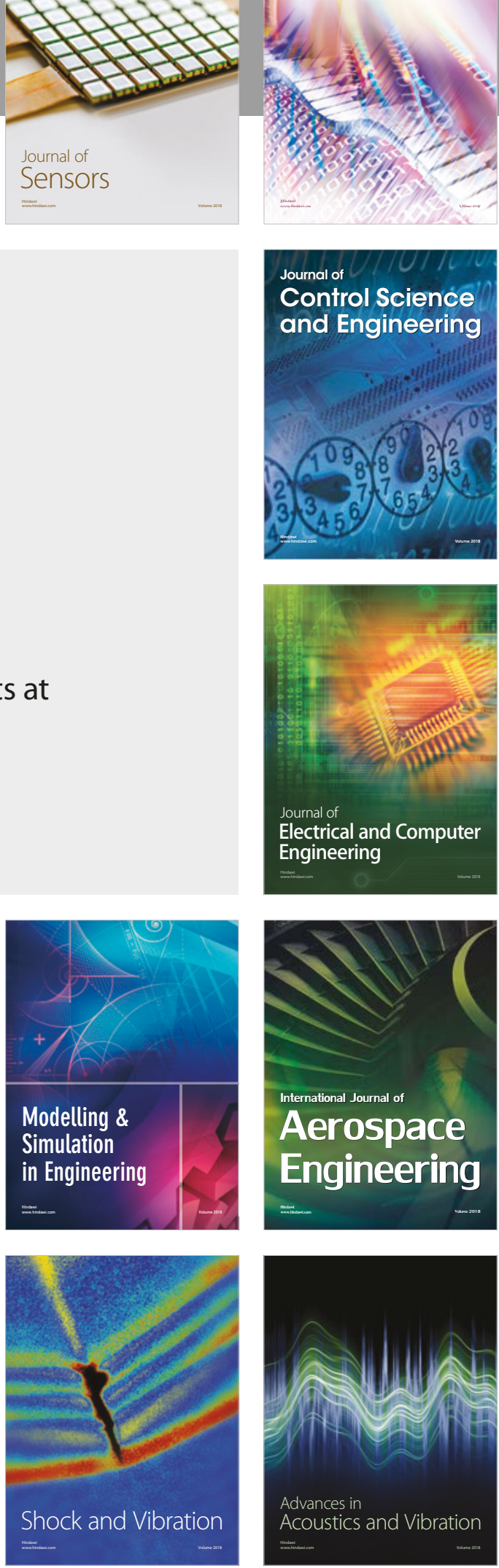